\title{
Hans-Joachim Hoppe
}

\section{Bulgarien}

Bulgarien stellt unter den mit Hitler-Deutschland verbundenen Ländem einen Sonderfall dar: trotz des Beitritts zum Dreimāchtepakt am 1. März 1941 konnte es sein Engagement auf ein geringes Ausmaß beschränken und sich weitgehende Eigenständigkeit bewahren. Seine Teilnahme am Kriege bestand selbst während des Balkanfeldzuges, in dessen Gefolge es Mazedonien und Thrazien gewann, im wesentlichen in Reserve- und Besatzerfunktionen. Mit Erfolg widerstand Bulgarien deutschen Forderungen, sich am Rußlandfeldzug - zumindest mit einer freiwilligen Truppe - zu beteiligen. Als einziges Achsenland unterhielt es bis kur vor dem Einmarsch der Roten Armee im September 1944 diplomatische Beziehungen zur Sowjetunion und wurde von Deutschen wie von Russen als ${ }_{n}$ Fenster" ${ }^{\text {zur Gegenseite geschätzt.1 }}$

$\mathrm{Zu}$ den Sonderfällen gehört auch das Schicksal der bulgarischen Juden, die den Krieg und insbesondere die kritische Phase im Frühjahr und Frühsommer 1943 trotz aller Restriktionen und Internierungen weitgehend unversehrt überstanden. Die Bulgaren sind stolz darauf, daß ihre jüdische Minderheit - zwischen 48000 und 51000 den Krieg als einzige im Machtbereich der Achse überlebte.2 Verfolgt und mit dem Tode bedroht wurden lediglich die Juden, die sich in der Widerstandsbewegung - bei den Partisanen - betātigten. Sie wurden jedoch nicht aus rassischen Gründen, sondern wegen staatsfeindlicher Betātigung inhaftiert und abgeurteilt. Laut bulgarischen Angaben waren 460 Personen jüdischer Herkunft aus politischen Gründen inhaftiert, darunter 29 mit Todesurteilen, von denen 17 vollstreckt wurden. Während des Krieges sind von 260 jüdischen Partisanen 125 ums Leben gekommen. Mehr als 1000 Personen waren aus politischen Gründen in Konzentrationslagern interniert, von denen zehn umkamen. Von offizieller Seite in Bulgarien wird immer wieder der hohe jüdische Anteil an der Widerstandsbewegung gewürdigt. Jüdische Widerständler traten im Kriege auch durch spektakuläre Attentate hervor, die heute von kommunistischer Seite heroisiert werden. ${ }^{3}$

Ein weniger rühmliches Kapitel stellt freilich die Politik Sofias gegenüber den Juden in den bulgarischen Besatzungsgebieten dar: im ehemals jugoslawischen Mazedonien und im ehemals griechischen Thrazien. Hier gab die bulgarische Führung den

1 Ausführlich Marshall Lee Miller, Bulgaria During the Second World War, Stanford 1974; Hans-Joachim Hoppe, Bulgarien - Hitlers eigenwilliger Verbündeter. Eine Fallstudie zur nationalsozialistischen Südosteuropapolitik, Stuttgart 1979. Die vorliegende Untersuchung basiert neben der Forschungsliteratur auf den einschlägigen Akten des Auswärtigen Amtes, auf umfangreichen Recherchen in der Zentralstelle in Ludwigsburg (Prozesse gegen Becketle, Bosshammer und von Hahn) sowie auf den bei Forschungsaufenthalten in Bulgarien gewonnenen Erkenntrissen.

2 Wolf Oschlies, Bulgariens Juden in Vergangenheit und Gegenwart Berichte des Bundesinstituts für ostwissenschaftliche und internationale Studien 16 (1972).

3 David Koen/Anri Assa, Die Rettung der Juden in Bulgarien 1941-1944, Sofia 1977. 
deutschen Wünschen nach, so daß alle Juden, ca. 12 000, aus diesen Gebieten deportiert wurden und im Vernichtungslager Treblinka umkamen. Auch in den neubulgarischen Gebieten wurden die Behörden aber erst aufgrund starken deutschen Drucks aktiv.

Die Zahlen über die in Bulgarien und in den besetzten Gebieten lebenden Juden variieren beträchtlich. Die Angaben über die aus Mazedonien und Thrazien deportierten Juden haben sich meist auf ca. 12000 eingependelt. Hingewiesen sei hierzu auf die Arbeiten von Raul Hilberg, Gerald Reitlinger, Ladislav Lipscher sowie auf das Gutachten Doerner und das Fauck-Gutachten des Instituts für Zeitgeschichte in München. ${ }^{4}$ Am gründlichsten und am stärksten auf Quellen gestützt ist die Untersuchung des Amerikaners Frederick B. Chary, The Bulgarian Jews and the Final Solution 1940 - 1944. Neben einer detaillierten Darstellung der Judenpolitik in Bulgarien finden sich bei Chary sorgfältige Berechnungen über die Zahl der deportierten und umgekommenen Juden der besetzten Gebiete. ${ }^{5}$ Die historische Entwicklung, das psychosoziale Umfeld und die politischen Hintergründe der bulgarischen Judenpolitik und das Verhältnis zwischen Juden und Bulgaren schildert sehr plastisch Wolf Oschlies. Seine Untersuchung gipfelt in der These von ${ }_{n}$ Bulgarien als dem Land ohne Antisemitismus", einer Charakteristik, der trotz aller Einwände zuzustimmen ist, denn Antisemitismus war in Bulgarien allenfalls eine Randerscheinung. In der Blütezeit des modernen Antisemitismus in Europa solidarisierten sich Politiker und Intellektuelle in Bulgarien mit ihren Juden. Und als der kleinen jüdischen Gemeinschaft Vernichtung drohte, traten Politiker - über alle Parteien hinweg - sowie breite Kreise der Öffentlichkeit für die Juden ein. 6

\section{Bulgariens Entwicklung in den dreißiger Jahren und im Zweiten Weltkrieg}

Bulgarien wurde seit 1935 von König Boris geführt; er regierte sein Land über von ihm ernannte Minister, die er aus Hofkreisen und Vertrauten reknutierte. Nach einem Offiziersputsch am 19. Mai 1934 installierte er eine Art königliche Diktatur mit Parteienverbot und ab 1938 mit einem Parlament mit begrenzten Vollmachten. Des Königs Macht war jedoch keineswegs absolut, und trotz ihrer Auflösung blieben die Parteien über ihre ehemaligen Mitglieder und Anhängerschaft ein wichtiger politischer Faktor: die Bauempartei, die (seit 1925 illegale) Kommunistische Partei, die bürgerlichen Parteien. Während der Königsdiktatur gab es drei politische Gruppierungen im Lande: 1. die illegale Opposition von Agrariern, Kommunisten und der elitāre Zirkel

4 Raul Hilberg, Die Vernichtung der europäischen Juden. Die Gesantgeschichte des Holocaust, Berlin 1982; Gerald Reitlinger, Die Endlösung. Frankfurt a.M. 1960'; Ladislav Lipscher, Die Verwirklichung der antijüdischen Malnahmen in den vom Dritten Reich beeinflußten Stanten, in: Karl Bosl (Hrsg.), Das Jahr 1941 in der europäischen Politik, München 1972, S. 121-141; Heinz Doerner, Gutachten über die Verfolgungsmaßnahmen gegen Angehörige der jüdischen Rasse“ in Rumānien, Ungarn und Jugoslawien während des 2. Weltkrieges und die Frage ihrer Veranlassung bew. Billigung durch deutsche Dienststellen, Hamburg 1955; Siegfried Fauck, Das deutsch-bulgarische Verhälnis 1939-1944 und seine Rückwirkung auf die bulgarische Judenpolitik, in: Gutachten des Instituts für Zeitgeschichte, Stuttgart 1966, Bd.2, S. 46-59.

5 Frederick B. Chary, The Bulgarian Jews and the Final Solution 1940-1944, Pittsburgh 1972.

6 Wolf Oschlies, Bulgarien - Land ohne Antisemitismus, Erlangen 1976. 
"Zveno" ("Kettenglied“), die im Exil oder im Untergrund agierten, 2. den Hof und die Regierungskreise, die allerdings in sich uneins waren, und schließlich 3. die legale Opposition der alten bürgerlichen Parteifunktionäre - Anwälte, Finanziers, Industrielle, Hāndler, Verwaltungsbeamte, die trotz des Parteienverbots immer noch Einfluß ausübten. Eine vierte Gruppe, die extreme Rechte, war ein Abklatsch kleiner faschistischer und nationalsozialistischer Parteien wie "Legionäre" und "Ratnici“, von denen nur die "National-soziale Bewegung" des Professors Alexander Cankov eine gewisse Bedeutung erlangte. Was den Gruppen an Anhang in der Bevölkerung fehlte, suchten sie durch Kontakte zu deutschen und italienischen Kreisen zu kompensieren. Der Rechten gelang es nicht, eine genuine faschistische Massenbewegung zu schaffen. ${ }^{7}$

Das Regime des Zaren Boris lehnte sich nicht an eine der faschistischen Gruppen an, sondern stützte sich auf persōnliche Autorität, auf Armee und Staatsapparat und auf eine breite Basis im Lande. Zur Erreichung der nationalen Ziele betrieb es eine Politik der friedlichen Verstāndigung mit den Mächten. Die deutsch-bulgarische Annāherung erfolgte in den dreißiger Jahren, zunāchst im Handel und im Rüstungssektor, dann im Hinblick auf die gemeinsamen Revisionsziele auch auf politischem Gebiet. Als Deutschland zunāchst Mitte 1938, dann verstärkt 1939 eine deutlichere Anlehnung Bulgariens an die Achse verlangte, erhoffte man bulgarischerseits neue Kredite und Unterstützung für seine Revisionswünsche: die Dobrudža und den Ägäiszugang. Doch bestātigte die bulgarische Führung nach Beginn des Zweiten Weltkrieges ihre "Neutralitāts- und Friedenspolitik “. Nach innen war Zar Boris bemüht, in der schwierigen Kriegszeit Regierung und Parlament unter fester Kontrolle zu halten. Durch den Kriegsverlauf wurde eine Option Bulgariens für die Achse - trotz abermaliger Bekräftigung der Neutralitāt durch die Regierung Filov im Frühjahr 1940 - fast unvermeidlich. Die Erwerbung der Süddobrudža mit deutscher Hilfe am 7. September 1940 verknüpfte Bulgarien enger mit dem Reich, so daß es eher geneigt war, auf deutsche Wünsche einzugehen. Angesichts des deutschen Aufmarsches in Rumānien, seiner Einbeziehung in die deutschen Kriegsplāne auf dem Balkan und des sowjetischen Drucks im Spātherbst 1940 suchte Bulgarien Anlehnung an Deutschland. Doch zögerte Bulgarien seinen Beitritt zum Dreimāchtepakt bis Frühjahr 1941 hinaus, bis seine Sicherheitswünsche sowie seine Revisionsansprüche weitgehend erfüllt waren.

In den Vorbereitungen zur Operation Marita (Feldzug gegen Griechenland) diente Bulgarien als Aufmarschbasis. Am 1. März 1941 trat Bulgarien dem Dreimāchtepakt bei - bei gleichzeitigem Einlaß deutscher Truppen; am 6. April marschierten diese von Bulgarien aus in Griechenland und Jugoslawien ein. Die Bulgaren nahmen am Feldzug selbst nicht teil, ihre Truppen dienten aber als Besatzer im Rücken der Wehrmacht und rückten Mitte April in Thrazien, Mazedonien und Pirot ein. Durch die Zustimmung zum deutschen Truppendurchmarsch und die Beteiligung an der Okkupation Griechenlands und Jugoslawiens wurde Bulgarien Kriegsteilnehmer auf deutscher Seite. Im Dezember 1941 erklärte Bulgarien auf deutschen Druck hin England und den USA ${ }_{m}$ symbolisch" den Krieg. Die UdSSR erklärte den Bulgaren am S. September 1944 den Krieg, um einen Vorwand zum Einmarsch und zur Absicherung des am 9. September zur Macht gelangten Regimes zu haben. ${ }^{8}$

\footnotetext{
7 Siehe dazu Ilčo Dimitrov, Bŭlgarska demokratǐ̌na obł̌testvenost, fał̌izmŭt i vojnata, 1934-1939, Sofija 1972.

B Detailliert Hoppe, Bulgarien.
} 


\section{Bulgarien und seine jüdische Minderheit}

Wegen der antisemitischen Politik des Dritten Reiches brachte die deutsch-bulgarische Allianz die jüdische Gemeinde in ernste Gefahr. Dabei entwickelte sich die Judenpolitik der bulgarischen Regierung ab September 1939 in enger Anlehnung an die deutsche, ab 1942 wurde sie für die jüdische Gemeinde angesichts des Beschlusses zur Endlösung existenzbedrohend. Während die Nationalsozialisten im Reich selbst und in den eroberten und besetzten Gebieten die Maßnahmen gegen die Juden mit Hilfe der SS ungehindert durchführen konnten, mußten sie in verbündeten Staaten das Auswärtige Amt und die diplomatischen Vertretungen einschalten. Dabei waren sie auf die Bereitwilligkeit der betreffenden Regierung angewiesen und mußten auf die Situation des betroffenen Landes Rücksicht nehmen.

Die bulgarische Gesellschaft war im allgemeinen gegenüber den Juden tolerant, die teils von im Mittelalter und am Ende des 15. Jahrhunderts aus Mitteleuropa und Osteuropa vertriebenen (deutsch sprechenden) Aschkenasim, zum größten Teil aber von 1492 aus Spanien und Portugal vertriebenen sephardischen "Spaniolen “ abstammten, vornehmlich in Sofia und anderen Stādten ansässig waren und ähnlich wie in der Türkenzeit durch kōniglichen Erlaß von 1890 eine Kultusgemeinschaft mit eigener Gerichtsbarkeit und Autonomie in religiōsen, zivilen und finanziellen Angelegenheiten bildeten, die dem bulgarischen Außenministerium, das offiziell „Ministerium für auswärtige Angelegenheiten und religiöse Kulte « hieß, unterstellt war. Die an sich kleine jüdische Minoritāt (1934 waren nur 0,8\% der Gesamtbevölkerung oder 48398 von ca. 6 Millionen Bulgaren Juden) ${ }^{9}$ trat lediglich in den Stādten in Erscheinung (in Sofia betrug der Anteil der Juden 10\%, das entsprach etwa der Hälfte aller bulgarischen Juden) und fiel gegenüber anderen Minoritāten - Türken und Griechen - nicht ins Gewicht (Anteil der Bulgaren an der Gesamtbevölkerung 84,4\%, d.h. 15,6\% Minoritāten, aber nur 0,8\% Juden). Nach einer Auflistung von Chary lebten 1940 in Bulgarien einschließlich in den seit August 1940 gewonnenen Gebieten (Dobrudža, Pirot, Thrazien und Mazedonien) 63403 Juden, davon in Sofia allein 27 289, in der Süddobrudža mit Dobric und Silistra 517, in Thrazien (Drama, Gjumjurdžina, Kavalla, Ksanti, Seres) 3975, in Mazedonien (Stip, Skopje, Bitola) 7230 und in Pirot (Ost-Serbien) 200 Juden. ${ }^{10}$

Die bulgarischen Juden waren weit weniger auffällig als die anderer Länder; als Städter waren ihr Bildungsstand und damit auch ihr Einkommen höher als das der bulgarischen Bevōlkerung, die hauptsāchlich auf dem Lande lebte. Die Juden arbeiteten vor allem im Handel und Handwerk, nur wenige besaßen großen Reichtum. Die jüdische Gemeinschaft bildete eine Enklave im bulgarischen Staat, doch gab es genügend Bindungen zwischen Juden und Bulgaren, viele Juden waren Mitglied in bulgarischen Berutsstānden, Kultur- und Sozialinstitutionen. Nur wenige errangen in der Armee und in der Gesellschaft hohe Posten. Politisch stimmte die jüdische Gemeinde meist mit der Regierung überein, abgesehen von den späten dreißiger Jahren, als die antisemitische Gesetzgebung begann. Die Juden traten nur selten in der Politik und in den Parteien hervor: im Gegensatz zu anderen kommunistischen Parteien Osteuropas

9 Zur Volkszählung von 1934 siehe Bulgarie, Annuaire Statistique, Jg. 27, 1935.

10 Detaillierte Angaben bei Chary, Bulgarian Jews, S. 204-207. 
hatte die bulgarische nur wenige Juden in ihrer Führungsspitze und auch keine eigene jüdische Sektion.

In Bulgarien nahm der Antisemitismus nie ein großes Ausmaß an, doch gab es ihn schon bei der Befreiung 1877/78 - wegen der antibulgarischen Politik Disraelis zur Zeit des Berliner Kongresses und wegen der protürkischen Haltung mancher Juden, die von einem bulgarischen Nationalstant weit weniger Toleranz gegenüber Minderheiten erwarteten als vom türkischen Vielvölkerstaat. Der bulgarische Haß richtete sich stärker gegen die Griechen, Türken und Muslimanen als gegen die Juden. Es gab sogar um die Jahrhundertwende in einigen bulgarischen Städten und Dörfern Judenpogrome, doch geschahen diese unter dem Eindruck der Pogrome im zaristischen Rußland und waren eher Auswüchse eines bāuerlichen Antisemitismus, dessen Ursprünge im Mittelalter zu finden sind und dem die ökonomische Basis fehlte - denn Geldverleiher waren nicht Juden (wie in Rumänien), sondern bulgarische Bankiers. Der moderne städtische Antisemitismus war bis in die späten zwanziger Jahre unbedeutend: Denn die bulgarische Nationalbewegung und die aus ihr entstandenen Parteien waren von liberalen Prinzipien und Traditionen inspiriert; als Sündenböcke dienten den Bulgaren eher Griechen und Türken als die Juden. Auch wurden die Minoritāten von den Bulgaren gut behandelt. Weder im Handel noch im Kampf um Arbeitsplätze bestand zwischen Bulgaren und Juden eine ernsthafte Konkurrenz.

Als der Antisemitismus in Europa wuchs, begannen die bulgarischen Intellektuellen über die Beziehung ihres Volkes zur jüdischen Gemeinschaft zu reflektieren und betonten - im Gegensatz zu anderen Ländem - das gute wechselseitige Verhältnis. So entstand der Mythos vom fehlenden Antisemitismus, der für die Einstellung der bulgarischen Intelligenz maßgebend wurde. 1937 publizierte ein jüdischer Joumalist namens Buko Piti ein Buch mit Stellungnahmen von ca. 150 führenden Persōnlichkeiten des öffentlichen Lebens gegen den Antisemitismus. 11 Vor dem Zweiten Weltkrieg gab es in Bulgarien keinen offiziellen Antisemitismus. Bis dahin unternahmen wohl die Rechtsextremisten einige antijüdische Aktionen und kleinere Pogrome, davon eines der aufsehenerregendsten im September 1939; doch die Regierung war darin nicht verwickelt und verhaftete sogar die Verantwortlichen. 12

\section{Die antijüdische Politik und Gesetzgebung der bulgarischen Regierung}

Im September 1939 erließ die Regierung Dekrete, die auslāndische Juden zum Verlassen des Landes verpflichteten. Kjoseivanov beauftragte Polizeichef Oberst Atanas Pantev mit der Ausweisung von $\mathbf{4 0 0 0}$ ausländischen Juden. Türkische und griechische Juden wurden unter Bewachung bis zur Grenze geschafft, doch die Masse der ausländischen Juden, meist aus Mitteleuropa vor den Nationalsozialisten geflohen, hoffte über Bulgarien nach Palästina zu gelangen. Einige zionistische Organisationen suchten Schiffe für deren Transport, als die Annāherung Bulgariens an Deutschland immer sichtbarer wurde. Pantev befahl den Juden, ihre Evakuierung auf Schiffen in Varna ab-

11 Buko Piti (Hrş.) Bǔlgarska obštestvenost za rasizma i antisemitizma, Sofija 1937.

12 New York Times, 28.9.1939, S. 6. 
zuwarten. Die Aktion hatte ein Jahr spāter einen tragischen Ausgang, als im Dezember 1940 die „Salvator ${ }^{\prime}$ - völlig überladen - bei der Durchfahrt durchs Marmarameer sank und ca. 200 Menschen den Tod fanden. Das Unglück wurde Gegenstand von Debatten im bulgarischen Parlament anläßlich der ersten antisemitischen Gesetzgebung, zugleich war es ein internationaler Skandal, so daß die anderen Schiffstransporte abgesagt wurden. Wegen der Schwierigkeiten der Einwanderung nach Palästina konnte Pantev seinen Auftrag nicht erfüllen und viele Juden blieben im Lande. ${ }^{13}$

Im Herbst 1939 hāuften sich antisemitische Auslassungen in der Presse und im Rundfunk - und auch seitens der Regienungsbeamten. Die Aufnahme des früheren Mitglieds der rechtsgerichteten „Ratnici“, Peter Gabrovski, in die Regienung im Oktober 1939 - zunächst als Eisenbahnminister und ab 15. Februar 1940 als Innenminister in der Regienung Filov - alarmierte die jüdische Gemeinde. Gabrovski holte seinen Protégé, das führende Mitglied der rechtsextremen "Ratnici“, Alexander Belev, ins Ministerium, der dort die juristische Sektion leitete, tatsāchlich aber als Experte für Judenfragen arbeitete, und andere "Ratnici“ als Mitarbeiter heranholte. Er unterhielt enge Beziehungen zu Berlin, um deutschen Wünschen nach Einführung antisemitischer Gesetze nach Nürnberger Vorbild nachzukommen. König Boris stimmte diesem Anliegen trotz Bedenken zu. Seinem Berater Ljubomir Christo Lulčev teilte er mit: „Es ist besser, wenn wir selbst solche Gesetze erlassen, als daß uns weitaus härtere von den Deutschen auferlegt werden. ${ }^{\mu 14}$

\section{Das „Gesetz zum Schutz der Nation“}

Im Sommer 1940 besuchte Judenkommissar ${ }^{\star}$ Belev Deutschland, um die Nümberger Gesetze zu studieren. Nach seiner Rückkehr kündigte Innenminister Gabrovski im Juli 1940 die Vorlage eines "Gesetzes zum Schutz der Nation“ an. Es sah die Registrierung aller Juden sowie wirtschaftliche und politische Restriktionen vor. Ausgenommen wurden Juden, die zum Christentum übergetreten, mit Bulgaren verheiratet oder im Militärdienst waren. Die Gesetzgebung lōste hitzige Debatten in der Öffentlichkeit der Hauptstadt aus. Mitglieder des bulgarischen Schriftstellerverbandes sandten Filov und den Parlamentspräsidenten einen Protestbrief, dem sich bald andere Gruppen wie der bulgarische Juristenverband, der Ärzteverband, der Heilige Synod, Studenten und Arbeiter anschlossen. Dimo Kazasov, prominentes Mitglied des Zveno, schickte einen offenen Brief an Filov. Es gab in Handel, Wirtschaft, rechtsextremen Kreisen, im Militär und in der Studentenschaft aber auch Befürworter der Gesetzgebung. In Artikeln und Broschüren wurde behauptet, die Juden hätten die Bulgaren wirtschaftlich ausgebeutet, in der Kriminalitāt stünden sie an der Spitze und gegenüber dem bulgarischen Staat seien sie illoyal. Angesichts der ihr unangenehmen öffentlichen Kontroverse peitschte die Sobranie das unter deutschem Druck entstandene unpopuläre „Gesetz zum Schutz der Nation" rasch nach nur dreitāgiger Debatte bei der ersten Lesung im November 1940 und einen Monat spāter in zwei Sitzungen, mit nur geringfügigen Änderungen durch. Für die Regierung geradezu peinlich waren

13 Chary, Bulgarian Jews, S. 36.

14 Laut Tagebuch-Eintragung des Zarenberaters Lulcev vom 11. November 1940. Chaim Oliver, Nie, spasenite, Sofije 1967, S. 64. 
die gegen das Freimaurertum gerichteten Passagen der Gesetzgebung, da ausgerechnet Ministerprāsident Filov und Innenminister Gabrovski ehemalige Freimaurer waren. Am 23. Januar wurde das Gesetz im bulgarischen Staatsanzeiger veröffentlicht. Der König hatte bewußt mit der Unterzeichnung bis nach dem bulgarischen Weihnachtsfest, dem 7. Januar 1941, gewartet, da das Gesetz auch nach seiner Auffassung dem Geist der Kirchenfeste widersprach. ${ }^{15}$ Nach der Publikation des Gesetzes kam es nun auf die praktische Anwendung an. Das Gesetz enthielt mehrere Fristen: In einem Monat sollten die Juden aus öffentlichen Ämtern ausscheiden, innerhalb von sechs Monaten einen Teil ihres Eigentums verlieren. Artikel 21 begrenzte den jüdischen Anteil im Schulwesen. Artikel 25 legte die Zahl der in bestimmten Berufen, im Handel und in der Industrie Tätigen fest, doch wurden die Bestimmungen großzügig gehandhabt. Und die in Mangelberufen Beschäftigten wie Ärzte, Zahnärzte etc. konnten bleiben; in Wirtschaft und Industrie wurden viele Juden über das Gesetz für zivile Mobilmachung auch weiterhin beschäftigt. Die Bestimmungen wurden von der Regierung lasch gehandhabt und oft unter Umgehung von Belevs Abteilung unterlaufen, so daß Extremisten behaupteten, das Gesetz diene nicht „zum Schutz der Nation“, sondern ${ }_{n}$ zum Schutz der Juden“. Die Konfiszierung jüdischen Eigentums, das man nach Artikel 26 des Gesetzes zunāchst registrierte, wurde dagegen strikt durchgeführt AuBerdem legte Finanzminister Dobri Božilov der jüdischen Gemeinde im Juli 1941 wegen "über 60 Jahre Ausbeutung Bulgariens" eine einmalige Sondersteuer auf. Das im Februar 1942 erlassene Gesetz gegen Landspekulation wurde gegen Juden besonders streng angewandt.

Die Regierung dehnte die Gesetzgebung nach dem Balkanfeldzug Ende April 1941 auch auf die besetzten Gebiete in Griechenland und Jugoslawien aus; diese Territorien waren annektiert worden, trotz des Berliner Vorbehalts einer endgültigen Regelung am Ende des Krieges. Aber in der Praxis behandelte Sofia die neuen, „kürzlich befreiten Territorien" als (fremde) besetzte Gebiete, die eine neue Verwaltungsspitze aus Bulgarien erhielten und im Parlament (anders als die 1940 erworbene Süddobrudža) nicht vertreten waren. Unter den Sondermaßnahmen für die neuen Provinzen sollte die zunāchst auf die Griechen beschränkte Emigrationserlaubnis für die Juden große Bedeutung erhalten.

In den eroberten Gebieten war die Voraussetzung für die Entwicklung einer Widerstandsbewegung, die von der „Vaterlāndischen Front“ organisiert wurde, am günstigsten. Da die Juden als Staatsfeinde definiert waren, wurden sie von der "Vaterlāndischen Front “ besonders umworben. Viele, vor allem jüngere Leute, schlossen sich den Partisanengruppen an. Etwa 70 jüdische Partisanen fielen dem Kampf zum Opfer. Von Juden wurden einige spektakuläre Widerstandsakte unternommen - wie die Sprengung des Benzindepots in Ruse im Oktober 1941. Der Anteil jüdischer Partisanen war mit 400 von 10000 (ca. 4\%) im Vergleich zum Bevölkerungsanteil sehr hoch. ${ }^{16}$

15 Der endgültige Gesetzestext ist veöffentlicht im Bulgarischen Stentsenzeiger: Düržaven vestnik, Nr. 16, 23. Januar 1941, S. 1-5; Auszüge in deutscher Sprache finden sich bei Oschlies, Bulgarien, S. 116-126; zur Diskussion und Verabschiedung des Gesetzes siehe Hoppe, Bulgarien, S. 93-96.

16 Siehe die Angaben in der Broschüre Evrei - zaginali v antifasistkata borba, Sofija 1958; detaillierter Nissan Oren, Bulgarian Communism. The Road to Power 1934-1944, New York 1971. 
Wahrscheinlich wären die Bulgaren auch in den folgenden Kriegsjahren nicht über die Bestimmungen des „Gesetzes zum Schutz der Nation“ hinausgegangen, wenn nicht der Druck von außen stärker geworden wäre: Wie überall in Europa wurde die Grundlage für die zweite Stufe der Judenpolitik in Berlin gelegt. Auf der WannseeKonferenz am 20. Januar 1942 in Berlin wurde die Deportation aller Juden aus ganz Europa und ihre Vernichtung ( ${ }_{n}$ Endlösung der Judenfrage") beschlossen. Wie in Rumänien und Ungarn konnten die deutschen Stellen aber auch im verbündeten Bulgarien nicht direkt agieren, sondern der Weg zur Deportation mußte vom Auswärtigen Amt bereitet werden; die Rolle des RSHA war hier mehr beratend als aktiv. Aus Bulgarien sollten die bulgarischen Juden, einschließlich der in den annektierten Gebieten lebenden, deportiert werden. Die bulgarische Regierung ging zumindest äußerlich sehr bereitwillig auf die deutschen Wünsche ein. So stimmte sie im Sommer $1941 \mathrm{der}$ Forderung zu, die mit den Bulgaren zusammen im Arbeitsdienst tātigen Juden (dem Kriegsministerium unterstellt) in speziellen $Z$ wangsarbeitsgruppen (dem Ministerium für öffentliche Arbeiten unterstellt) zusammenzufassen. ${ }^{17}$

Im Juni 1942 forderte Unterstaatssekretār Martin Luther vom Auswärtigen Amt die bulgarische Regierung auf, die Rechte der in Deutschland und im Protektorat lebenden bulgarischen Juden abzuerkennen, damit sie mit den anderen Juden in Deutschland deportiert werden könnten. Sofia stimmte zu, behielt sich aber vor, eine Liste der betreffenden Juden aufzustellen. Außerdem forderte es, um einen Alleingang zu vermeiden, ähnliche Vereinbarungen mit anderen Staaten, nämlich mit Rumänien und Ungarn.18 Die Durchführung der deutschen Pläne machte zunächst eine Änderung und Verschärfung der bulgarischen Judengesetze erforderlich. Die deutschen Stellen waren unzufrieden mit der bulgarischen Judendefinition und den zahlreichen Ausnahmen des Staatsschutzgesetzes. Das bulgarische Gesetz war in erster Linie auf die Religion abgestellt, so daß alle Juden, die bis zum 1. September 1940 getauft waren, vom Gesetz ausgenommen blieben, gleichgültig, ob sie mit einem Christen verheiratet waren oder nicht. Nicht betroffen waren auch Juden, die vor dem 23. Januar 1941 (Eriaß des Gesetzes) Bulgaren geheiratet hatten. Kriegsveteranen, Invaliden, Witwen und Kriegswaisen blieben ebenfalls von den Restriktionen weitgehend verschont. Auch fehlten in den Bestimmungen Mischlingskategorien wie in den Nürnberger Gesetzen.

\section{Das „Kommissariat für Judenfragen“ (KEV)}

Während die Wannsee-Konferenz tagte, reiste Belev auf Weisung Gabrovskis nach Berlin, um die neuesten Entwicklungen in der Judenfrage zu erkunden. Er berichtete im Juni 1942, daß auch die bulgarische Regierung Vorbereitungen zur Judendeporta-

17 Telegramme des deutschen Gesandten Beckerle an das Auswärtige Amt vom 24. und 31. Juli 1941 (178874 und 178886), in: PAA, Abteilung Inland, Inland II $\mathrm{B}$, Bulgarien, Bd. 2.

18 Zur Judenpolitik in Bulgarien siche insbesondere den Aktenband Inland II g. Judentrage in Bulgarien (1941-1944), Bd. 183, im Politischen Archiv. Zur Behandlung der in Deutschland und im Protektorat lebenden bulgarischen Juden siehe den Telegrammwechsel zwischen Unterstaatssekretär Luther und dem Gesandten Beckerle: Luther an Beckerle, 19. Juni 1942 (486203-205), als Anlage dazu der Entwurf eines deutsch-bulgarischen Abkommens über jüdische Bürger (486206-207); Beckerle an $A$, 6. Juli 1942 (486208-209); Luther an Beckerle, 5. August 1942 (486211); Bericht Beckerles an AA, 9. Juli 1942 (486216) mit Anlage Verbalnote des Bulgarischen Außenministeriums vom 7. Juli 1942 (486217). 
tion und Maßnahmen zur vollstāndigen Konfiszierung des jüdischen Eigentums treffen müsse. Noch im gleichen Monat brachte die Regierung eine Vorlage im Parlament ein, die den Ministerrat ermāchtigen sollte, Maßnahmen zur Lösung der Judenfrage zu ergreifen. Durch das Regierungsdekret vom 26. August 1942 wurde ein Kommissariat für jüdische Fragen eingerichtet (Komisarstvo za evreiskite vuprosi - KEV), das für alle Maßnahmen gegen die Juden zuständig war, ausgenommen diejenigen, die unter das Antispekulationsgesetz und das Judenbesteuerungsgesetz fielen und deshalb Angelegenheit des Finanzministeriums blieben. Das Dekret änderte die Definition des "Juden", in die zu den religiösen auch rassische Elemente aufgenommen wurden, reduzierte die Ausnahmekategorien und legte die rechtliche Grundlage für Deportationen.

Kern des Gesetzes war der Artikel 29, der die „Aussiedlung “ der Juden Sofias „in die Provinzen oder außerhalb des Königreichs“ vorsah. Alle arbeitslosen Juden sollten die Stadt bis zum 1. November 1942 verlassen haben. Das Kommissariat entfernte die Judenfrage aus dem Blickpunkt der Öffentlichkeit, hielt das Parlament aus der Angelegenheit heraus und befreite den Kōnig von der Pflicht, spektakuläre Judenmaßnahmen selbst abzeichnen zu müssen. Das Parlament billigte neben weiteren Erlassen zur Judenfrage das Dekret vom 26. August im September 1942.19 In den nāchsten zwei Jahren war das Kommissariat (seit dem 18. November 1942 mit stāndigem Sitz am Boulevard Dondukov) das Hauptinstrument zur Durchsetzung der Judengesetzgebung. Nach seiner Emennung übernahm Belev seine bisherigen Mitarbeiter aus dem Eisenbahnministerium in sein Kommissariat, viele davon waren Ratnici und Antisemiten, die meisten jedoch Staatsbedienstete, die von den hohen Gehältern angelockt wurden oder die Möglichkeit zu illegalen Einnahmen sahen. Wāhrend der Hauptaktionen im Frühjahr 1943 waren in seinem Amt über 100 stāndige und fast 60 zeitweilige Mitarbeiter beschäftigt. Das Budget des Kommissariats wurde hauptsāchlich von jüdischen Bankkonten, Steuem und anderen Abgaben finanziert. Anfangs stieß Belev mit seinen Maßnahmen auf Schwierigkeiten und wurde selbst von Regierungsmitgliedern übergangen: So hob Filov die Bestimmung über das Tragen von Judenstemen für in Mischehen lebende Juden und schließlich, auf Protest des Metropoliten von Sofia, auch für die zum Christentum konvertierten Juden auf. Auch das Justizministerium schritt gegen einige Beschlüsse Belevs ein. Das RSHA in Berlin bemängelte, daß das Tragen von Judensternen und die Markierung der Häuser lasch gehandhabt werde. Wegen Produktionsschwierigkeiten standen nicht genug Steme zur Verfügung, so daß nur 20\% der vorgesehenen Quote erfüllt war. Viele Juden hielten sich nicht an die Anordnung, andere trugen angesichts der Sympathiebekundungen ihrer bulgarischen Nachbarn die Sterne mit Stolz und hefteten sich neben den Sternen Abzeichen mit Bildern des Königs und der Kōnigin an. Die Deutschen führten das „arrogante Verhalten" der Juden auf die Indifferenz der bulgarischen Bevölkerung zurück. Zudem protestierten Rumānien, Ungarn, Frankreich, Spanien und Italien gegen die Anwendung des Erlasses auf ihre in Bulgarien lebenden jüdischen Staatsbürger. Italien richtete über $\mathbf{4 0 0}$ Noten an das Außenministerium und erlaubte vielen Juden den Erwerb der italienischen Staatsbürgerschaft und die Ubersiedlung nach Italien. Das bulgarische Außenministerium pflegte diese Noten mit der Anweisung zur Milderung der 
Regelungen an Belev weiterzuleiten. ${ }^{20}$ Doch bald setzte das Kommissariat eine stärkere Beachtung der Bestimmungen durch: Bereits Ende Dezember 1942 standen ausreichend Judensterne und Kennzeichen zur Verfügung; Kontrollen wurden durchgeführt und bei Nichtbeachtung der Bestimmungen Strafen verhängt.

Eine Hauptaufgabe des Kommissariats war die Registrierung aller Juden. Nach mehr oder weniger zuverlässigen Statistiken lebten 1942 unter bulgarischer Verwaltung und fielen damit in die Zustandigkeit des KEV 63400 Juden, davon 51500 (3000 mehr als beim Zensus von 1934) in Altbulgarien, 500 in der Süddobrudža, 4000 in Westthrazien, 7200 in Mazedonien und 200 in Pirot. Für die spātere Behandlung gab die Tatsache den Ausschlag, daß die in der Dobrudža lebenden Juden bulgarische Staatsbürger waren, die in den okkupierten Gebieten hingegen nicht. Die Juden wurden in bestimmten Wohngebieten (praktisch Ghettos) zusammengefaßt: in Sofia im westlich vom Zentrum liegenden Armenviertel nuč Bunar“ (Drei Brunnen). Die Konsistorien wurden dem Kommissariat unterstellt. Bis Ende Mărz 1943 mußten 680 Familien (1904 Personen) Sofia verlassen und in der Provinz bei anderen jüdischen Familien unterkommen. 21

Das Kommissariat plante, nach der Registrierung, alle Juden zu deportieren und ihr Eigentum zu konfiszieren: Nach Schätzungen auf der Grundlage von 23000 Steuererklärungen belief sich das jüdische Eigentum auf ca. 7,5 Mrd. Leva (91 Mill. US-Dollar), die Einheitssteuer betrug 1,7 Mrd. Leva (21 Mill. US-Dollar), d.h. 20-25\% des jüdischen Eigentums. Die Juden in den besetzten Territorien zahlten 300 Mill. Leva (3,6 Mill. US-Dollar) Sondersteuer, bei einem Gesamtvermögen von ca. 1,5 Mrd. Leva (18,2 Mill. US-Dollar). ${ }^{22}$ Von den Juden der besetzten Gebiete, die deportiert wurden, wurde alles Eigentum konfisziert; das Vermögen bis auf das Existenzminimum fiel dem Kommissariat zu. Alle Gelder und Wertgegenstānde mußten auf Sperrkonten deponiert werden. Sachwerte wie Geschirr, Gemālde, Briefmarkensammlungen, Orientteppiche, Musikinstrumente wurden registriert und dem Kommissariat zur Disposition gestellt. Von ihren Konten durften die Juden monatlich hōchstens 6000 Leva (72,80 US-Dollar) pro Familie für den persönlichen Gebrauch abheben; Eigentum über 10000 Leva (121 US-Dollar) im Jahr durften sie nur mit besonderer Erlaubnis verkaufen. Bis 3. Màrz 1943 belief sich der Wert der eingefrorenen jüdischen Konten auf über 801 Mill. Leva (9,7 Mill. US-Dollar). Nach Schātzungen betrug der Wert des konfiszierten jüdischen Eigentums ca. 4,5 Mrd. Leva (54,7 Mill. US-Dollar) - nach dem Stand von 1939.23

Weitere Restriktionen schlossen die Juden aus der bulgarischen Gesellschaft aus: Verbot des Besitzes von Radios und Telefonen, Konfiszierung aller Automobile, Motorrāder und Fahrräder, Einschrānkung der Bewegungsfreiheit, der Geschäftstātigkeit und der Ausübung bestimmter Berufe, Ausschluß der Juden von allen bulgarischen Schulen mit numerus clausus, Festsetzung von Quoten für die übrigen Schulen, strenge Handhabung der Kriegsrationierung, Verbot der wenigen noch existierenden

20 Siehe Bericht Schellenbergs (RSHA) an Luther (AA), 21. November 1942 (486243-248) und Steliungnahme Beckerles zum RSHA-Bericht vom 14. Dezember 1942 (486274-277).

21 Chary, Bulgarian Jews, S. 58-61.

22 Detailliert Benjamin Arditi, Les Juifs de Bulgarie sous le Regime Nazi 1940-1944, OJ. (hebr. Original: Tel Aviv 1962); David B. Koen, Ekspropriacijata na evrejskite imuštestva prez perioda na Hitleristkata okupacija, in: Godisnik 2 (1967), S. $109 \mathrm{ff}$.

23 Siehe Koen, Ekspropriacijata, passim. 
jüdischen Organisationen, Schließung von Büchereien und Verlagen, Kontrolle der jüdischen Wirtschaftsunternehmen wie Volksbanken, Kreditinstitute, Versicherungsgesellschaften durch das Kommissariat.

Die jüdischen Arbeitsdienstgruppen, die zunāchst Teil des allgemeinen Arbeitsdienstes für alle bulgarischen Jugendlichen waren, wurden auf deutsches Verlangen vom allgemeinen Arbeitsdienst getrennt und ausschließlich bulgarischen Offizieren unterstellt, wodurch die Dienstverpflichtung für die Juden den Charakter des Strafdienstes statt wie für die Bulgaren den einer „nationalen Ehrenpflicht“ erhielt. Schon vor dem August-Dekret hatte das Kabinett am 19. Juli 1942 die Einziehung von jüdischen Männern von 20 bis 45 Jahren zum Arbeitsdienst angeordnet, sie wurden im Straßen- und Eisenbahnbau in strategisch wichtigen Gegenden Bulgariens eingesetzt.

\section{Die Vorbereitung der Judendeportationen}

Die Hauptaufgabe des Kommissariats war die Vorbereitung der Judendeportationen aus Bulgarien und den besetzten Gebieten als Teil der „Endlösung“. Als erstes stimmte die bulgarische Regienung im Juli 1942 der Deportation der im Deutschen Reich lebenden bulgarischen Juden zu.24 Zur selben Zeit schlug das Auswärtige Amt die Deportation der Juden unter bulgarischer Verwaltung vor, aber das RSHA bat wegen anderweitiger Aktionen um Verschiebung auf das Jahr 1943. Doch sollte auf diplomatischem Wege das Terrain für die Aktion vorbereitet werden.25 Zweifel an der bulgarischen Einstellung in der Judenfrage löste im Juli 1942 ein „Skandal“ aus. Der Präsident des jüdischen Zentralkonsistoriums, Josef Geron, hatte dem Kōnig aus Anlaß des 5. Geburtstages des Kronprinzen Simeon (geboren am 16. Juni 1937) einen Glückwunsch geschickt, worauf der König ein Dankestelegramm an Geron und die jüdische Gemeinde richtete. Glückwünsche und Telegramme dieser Art waren nichts außergewöhnliches, doch erregten sie diesmal durch den Zeitpunkt Aufsehen - nämlich einen Monat nach Verabschiedung des Ermāchtigungsgesetzes zur Anti-JudenPolitik und kurz vor Veröffentlichung des Dekrets vom August 1942. Zur Verärgerung der deutschen Stellen unterhielt König Boris auch noch danach Kontakt zur jüdischen Gemeinde und unterzog sich persönlich der unangenehmen Aufgabe, dem Oberrabbiner von Sofia Asher Hananel das Gesetz vom August zu erläutern. 26

Im September 1942 intervenierte die bulgarische Regierung wegen der Behandlung ihrer jüdischen Bürger in Frankreich und anderen von Deutschland besetzten Gebieten. ${ }^{27}$ Im Oktober 1942 hielt Luther nach einer Unterredung mit Beckerle die Bulgaren für reif, auch ihre Juden zu deportieren, und bot ihnen die Dienste des Reiches an. Luther wies Beckerle an, von den Bulgaren wollte man ein Kopfgeld von 250. - RM pro deportierter Person zur Deckung der deutschen Ausgaben verlangen; man war allerdings bereit, über diesen Posten zu verhandeln, um den Bulgaren keinen Vorwand zur Ablehnung der Aktion zu liefern. Die deportierten Juden sollten beim Verlassen

24 Beckerte an AA, Sofia 9. Juli 1942 (486208-209).

25 Chary, Bulgarian Jews, S. 69.

26 Ebenda, S. 70.

27 Ebenda, S. 711 . 
ihres Landes die bulgarische Staatsangehörigkeit verlieren. ${ }^{28}$ Die Bulgaren reagierten ausweichend. So berichtete Beckerle, daß die Bulgaren die Juden dringend zum StraBen- und Eisenbahnbau benötigten. Auch bestünde im Kabinett noch keine einheitliche Meinung über die Deportationen. ${ }^{29}$ In einem Bericht vom 21 . November 1942 brachte Walter Schellenberg, Chef des Auslandsnachrichtendienstes im RSHA, seine Enttäuschung über die bulgarische Haltung zur Judenfrage zum Ausdruck. ${ }^{30}$ Nach den strengen Maßnahmen vom August 1942 und der Einrichtung des Kommissariats sei die bulgarische Bevölkerung zu der Ansicht gelangt, daß die antijüdischen Gesetze zu weit gingen, $\mathrm{da} \beta$ die Lage der Juden gebessert und daß Belev Hindernisse in den Weg gelegt werden müßten. Auch sei der jüdische Einfluß am Hofe und in der Politik durch Verwandtschaftsbeziehungen groß. Innenminister Gabrovski wurde Laschheit in der Judenfrage vorgeworfen. So seien am 27. September 1942300 Juden zum Innenministerium marschiert und hätten ihm eine Petition mit Protesten gegen die neuen antisemitischen Bestimmungen überreicht. Gabrovski habe die Gruppe im Hof des Ministeriums empfangen und ihr versichert, das Schlimmste sei schon vorbei. Diese Haltung habe die Juden ermutigt, sich gegen Belevs Anordnungen zu wehren. Gabrovski habe Ende September der Presse aus Furcht vor der öffentlichen Meinung die Berichterstattung über die neuen Maßnahmen verboten. Und Belev habe er den Wunsch des Hofes und der Regierung nach einer Milderung der Judenpolitik mitgeteilt. Der deutsche Gesandte schwāchte den Bericht Schellenbergs ab: die Bevölkerung sei an Judenmaßnahmen desinteressiert, die Regierung handhabe lediglich die ökonomischen Maßnahmen gegen die Juden streng. Er dementierte die Schilderung Schellenbergs über Gabrovski; dieser habe von der Demonstration vor dem Innenministerium nichts gewußt. Ob er eine Petition entgegengenommen habe, wisse er nicht. Zur Judenkennzeichnung meinte Beckerle, nur die italienischen Juden seien von der Regelung ausgeschlossen. Der Petition der Metropoliten messe die Regierung keine Bedeutung zu. 31

In einer Verbalnote vom 2. November 1942 teilte das bulgarische Außenministerium der Reichregierung mith daß es grundsātzlich der Deportation der Juden zustimme, diese jedoch zur Zeit für öffentliche Arbeiten in Bulgarien noch dringend benōtigt würden. Das Angebot der Reichsregierung zur Entsendung eines Judenberaters nahm es dankend an. Außerdem forderte es, daß die bulgarischen Deportationen mit denen aus Rumānien gekoppelt werden sollten, da Bulgarien nicht in der Lage sei, auch nur einen Teil der Juden allein zu deportieren. Die Verbalnote bezeichnete den Preis von 250.- RM Kopfgeld als zu hoch, denn das jüdische Vermögen werde zur Unterstützung der bulgarischen Wirtschaft benötigt ${ }^{32}$ Im Deutschland-Referat des Auswärtigen Amtes war man der Ansicht, die Verhandlungen sollten nicht mit der Preisfrage belastet werden, um den Bulgaren keinen Vorwand für eine Verzögerung der Judendeportationen zu liefern, doch hielt man ein Kopfgeld von 100.- RM für das absolute Minimum. ${ }^{33}$

28 Aufzeichnung Luthers, 9. Oktober 1942 (486236); Luther an Beckerle, 15. Oktober 1942 (486234-235).

29 Telegramm Beckeries, 2. November 1942 (486237).

30 Schellenberg an Luther, 21 . November 1942 (486242-248).

31 Bericht Beckerles an AA, 14. Dezember 1942 (486242-248).

32 Beckerle an AA, 16. November 1942 (486261); Verbalnote des Bulgarischen Außenministeriums vom 2. November 1942 (486262-263).

33 Rademacher an Müller, 19. März 1943 (486264); Klingenfuß, 4. Dezember 1942 (486272). 
Als Judenberater" wurde Theodor Dannecker nach Sofia entsandt. Seine Aufgaben in Bulgarien sollte er in neun Monaten erledigen. In Sofia wurde Dannecker dem Polizeiattaché Karl Hoffmann zugeteilt. Von Dezember 1942 bis Februar 1943 arbeitete Dannecker zusammen mit Belev die Deportationsplāne aus. Beckerle informierte Berlin über die Schwierigkeiten in der Haltung Bulgariens zur Judenfrage, die deshalb bestünden, weil die Juden in Bulgarien Arbeiter seien und die Existenz anderer Minoritäten in Bulgarien ein Verständnis für die Judenfrage verhindere. ${ }^{34}$ Wāhrend man auf höherer Ebene noch strith, kam das Kommissariat mit den RSHA-Vertretern zu vollem Konsens über die praktische Durchführung der Deportationen: Ungefähr 10000 bis 20000 Juden sollten von Thrazien (Belomorie) und Mazedonien zusammen mit "unerwünschten“ Juden aus Altbulgarien abtransportiert werden. Die letzteren würden mit Verlassen des Königreiches ihre bulgarische Staatsbürgerschaft verlieren. Die bulgarischen Juden sollten erst zu einem spāteren Zeitpunkt deportiert und zunāchst in Arbeitslagern zusammengefaßt werden. 35

Dannecker beklagte in seinem Bericht an Eichmann die lasche Durchführung der antijüdischen Bestimmungen: In Bulgarien ansāssige Juden aus Deutschland, Polen, dem Protektorat und Österreich konnten sich retten, indem sie italienische, spanische und portugiesische Staatsbürger wurden. Viele sephardische Juden erhielten die spanische Staatsbürgerschaft, Juden aus Mazedonien und Thrazien in der Nāhe des Interessengebietes Italiens die italienische. ${ }^{36}$ Belev suchte die Planungen schnell durchzuführen, obwohl das Kabinett noch nicht über die Deportationen der bulgarischen Juden entschieden hatte. Bereits am 3. Februar erteilte er seinen Repräsentanten in allen Teilen Bulgariens den Auftrag, Listen der in ihrem Bezirk lebenden Juden anzufertigen und diese bis zum 9. Februar ans Kommissariat zu schicken. Der administrative Leiter Jaroslav Kalicin befaßte sich bereits mit konkreten Maßnahmen zur Durchführung der Deportationen. 37

Am 22. Februar 1943 unterzeichneten Belev und Dannecker ein Abkommen über die Deportation von 20000 Juden. ${ }^{38}$ Das Abkommen sah insgesamt sechs Deportationszentren in Bulgarien und den besetzten Gebieten vor, nämlich Skopje, Bitola, Pirot, Gorna Džumaja, Dupnica und Radomir. Bulgarien sollte die Kosten tragen. Juden in Mischehen sollten nicht deportiert werden. In Punkt 8 des Abkommens hieß es makaber: „In keinem Fall wird die bulgarische Regierung die Rückkehr der deportierten Juden verlangen. “

Dannecker informierte noch am selben Tag Eichmann. ${ }^{39}$ Laut Belev sollten alle Juden von Thrazien und Mazedonien und eine bestimmte Anzahl von Juden aus den alten Gebieten, alles in allem 20000 Menschen, spätestens bis Ende Mai 1943 deportiert werden: aus Mazedonien und Pirot 8000 Juden, aus Thrazien 6000 und aus den

34 Otto von Hahn (Abteilung Deutschland) an Personalabteilung des AA, 2. Januar 1943 (486268); RSHA an Luther, 10. Dezember 1942 (486270-271). Telegramm Beckerles an AA vom 22. Januar und 10. Februar 1943 (486278-280, 486290).

35 Belev an Gabrovski, 4. Februar 1943, in: Natan Grinberg, Dokumenti, Sofija 1945, S. 8-11. Bericht Dannekkers an Eichmann, 8. Februar 1943 (486285-287), gesehen von Beckerle.

36 Dannecker an Eichmann, 8. Februar 1943 (486288-289), gesehen von Beckerle.

37 Dannecker an Eichmann, 16. Februar 1943 (486293-294); Grinberg. Dokumenti, S. 11 ff.

38 Kopien der bulgarischen Version des Dannecker-Belev-Abkommens liegen in der Hebriischen Sektion des Balkanistik-Instituts der Bulgarischen Akademie der Wissenschaften. Eine Kopie ist abgebildet in: Grinberg, Dokumenti, S. $14 \mathrm{ff}$. Siehe hier die Anlagen $A 1$ bis As.

39 Dannecker an Eichmann, 23. Februar 1943 (486296-299). 
alten Territorien ebenfalls 6000 . Zunāchst sollten Anfang März die thrazischen Juden zu den Transportstationen geschickt werden, darauf die bulgarischen und mazedonischen Juden. Die Deportationen nach Polen sollten aus allen sechs Abfahrtszentren Ende März beginnen. Zur Vorbereitung und Durchführung der Aktion erhielten die KEV-Mitarbeiter entsprechende Instruktionen. $40 \mathrm{Kraft}$ des Ermächtigungsgesetzes beschloß das Kabinett am 2. März 1943 auf der Grundlage von Belevs Vorschlägen eine Reihe von Deportationsdekreten wie die Abstellung von Personal, den kostenlosen Transport, den Verlust der Staatsbürgerschaft, die Konfiszierung des jüdischen Eigentums.

Alle Dekrete dienten der Ausführung des Erlasses Nr. 127, der dem Kommissariat auftrug, „über die Grenzen des Landes gemāß der Ubereinkunft mit den deutschen Behörden bis zu 20000 Juden zu deportieren, die die kürzlich befreiten Territorien bewohnen“.41 Wichtige Konsequenzen sollte die Einschränkung des Erlasses auf "kürzlich befreite Territorien" haben. Doch wurde im Innenministerium auch erwogen, mit thrazischen und mazedonischen Juden die „Unerwünschten“" (neželatelni) aus Albulgarien zu deportieren. So hatte Belev offenbar in dem Abkommen mit Dannekker nachträglich die einschränkende Formulierung waus den neuen bulgarischen Gebieten Thrazien und Mazedonien" gestrichen, was nur die Einbeziehung von Juden aus Altbulgarien in die Deportationsplāne bedeuten konnte, denn in den besetzten Gebieten lebten nicht mehr als ca 12000 Juden. Die Regierung hatte in Thrazien und Mazedonien freie Hand, bei der Deportation der bulgarischen Juden mußte sie dagegen mit mehr Widerständen in der Öfentlichkeit rechnen, obwohl die Operationen unter strenger Geheimhaltung erfolgen sollten. Zweifellos betrachtete die Regienung die neuen Gebiete mit besonderer Aufmerksamkeit und war bemüht, in diesen Territorien den Anteil der nichtslawischen Bevölkerung zu vermindern. Diesem Ziel entsprachen die Deportationen der fremden Juden, nachdem sie bereits die Griechen zur Abwanderung ermuntert hatten.42

Laut Deportationsplan des Kommissariats wurde das Königreich in fünf Gebiete eingeteilt: Mazedonien, Thrazien, Pirot, den Bezirk Sofia und Restbulgarien. Alle Juden der ersten vier Gebiete, die Stadt Sofia ausgenommen, sollten deportiert werden, die thrazischen Juden zuerst, dann die aus Altbulgarien, aus Pirot und Mazedonien. Während aus den besetzten Gebieten alle Juden abtransportiert werden sollten, bereitete das Kommissariat eine Selektion aus Altbulgarien vor. Ausgenommen waren die Städte in Südwestbulgarien, aus denen wegen ihrer strategischen Bedeutung und der Nähe zu den besetzten Gebieten die gesamte jüdische Bevölkerung deportiert werden sollte. So wurden die KEV-Vertreter und Distriktgouverneure in Altbulgarien einschließlich der Bezirke Sofia und Vraca am 22. und 23. Februar aufgefordert, dem Kommissariat innerhalb von 24 Stunden Listen von allen Juden zu übersenden, die "reich, prominent und allgemein gut bekannt", als "Reprāsentanten jüdischen Geistes“ oder als "Anhānger staatsfeindlicher Ideen “ hervorgetreten seien. Dahinter stand die Absicht, Agitatoren auszuschalten und die jüdische Elite zu dezimieren. Die Auf-

40 Grinberg, Dokumenti, S. 16-23.

11 Chary, Bulgarian Jews, S. 211.

42 Aufzeichnungen Woermanns vom 18. Februar und 29. Mär 1943 (173822, 173871-878), in: PA, Büro Stantsseletetir Bulgarien, Bd. S. Die Bulgaren wollten die Griechen in die deutsche Besatzungszone in Griechenland abschieben, doch erlaubuen die deutschen Behörden dies nicht. 
stellung der Listen und noch mehr die darauf folgende Selektion war recht subjektiv und willkürlich, Belev erhielt die Namen von ca. 9000 Juden (3000 Familien), von denen er 8400 auswāhlte. ${ }^{43}$

Mit der bulgarischen Staatsbahn war schon der Transport von ca. 5800 Juden aus verschiedenen Gegenden des Königreichs am 10. und 11. März nach Pirot und Radomir vereinbart. Am 8. Mārz sandte Belev die Namen der selektierten Juden an die Regionalpolizei mit der Anweisung zu ihrer Arretierung. Aus Südwestbulgarien, wo auch die Abfahrtszentren lagen, sollten alle Juden deportiert werden. In Dupnica wurden zwischen dem 25. Februar und 2. März alle Juden unter Hausarrest gestellt; auch in Goma Dzumaja und Kjustendil lief die Aktion an. Am 10. März war in Radomir, Dupnica, Gorna Dzumaja und Pirot alles zur Aufnahme der bulgarischen Juden vorbereitet. Belevs Judenlisten überschritten sogar die im Abkommen vorgesehene Zahl um 2500; statt der geplanten 6000 kam er auf 8500. Dafür waren in Mazedonien und Thrazien nur 11500 Juden zur Deportation vorgesehen, so daß doch die Gesamtzahl von 20000 nicht überschritten wurde. Langfristig aber war geplant, alle Juden aus Bulgarien zu deportieren. 44

\section{Das Scheitern der Deportation der bulgarischen Juden:}

\section{Die Pešev-Aktion}

Die Plāne des KEV blieben nicht geheim. Als die Polizei die Juden in Plovdiv am 10. Mārz 1943 zusammentrieb, reiste Dimitur Stankov, ein prominenter Bürger, nach Sofia, um bei Filov vorzusprechen. Kiril, der Bischof von Plovdiv und spätere Patriarch von Bulgarien, richtete ein Protestschreiben an den König. Der Heilige Synod sandte einen weiteren Protest. Der Präsident des bulgarischen Schriftstellerverbandes Stiljan Cilingirov und ein anderer berühmter Schriftsteller intervenierten beim König und beim Premier Filov zugunsten der Juden. Am wirksamsten aber waren die Aktionen der Juden selbst: Prominente Juden in Sofia erfuhren von den Deportationsplānen aus verschiedenen Quellen, z.B. von Liljana Panica, der Sekretārin Belevs! Juden aus Kjustendil waren vom Kommissariatsarzt Vatev und vom Distriktgouverneur Ljuben Mitenov informiert worden. Sie baten einflußreiche bulgarische Freunde, für sie in der Hauptstadt zu intervenieren und sammelten Gelder zur Bestechung von Kommissariatsmitarbeitern. Am 4. Mārz erhielten die Juden von Kjustendil Ausgehverbot, am 10. Màrz sollten sie in das Femandes-Tabaklager geschafft und von dort nach Radomir transportiert werden. Eine Delegation von fünf prominenten Bürgern aus Kjustendil beschloß, nach Sofia zu reisen und dort bei der Regienung wegen der Juden vorzusprechen. Großen Einfluß hatte auch Jako Baruh, ein Mitglied des illegalen zionistischen Zentrums, der Visa nach Palästina beschaffte. Paradoxerweise wandten sich ausgerechnet Regierungsmitglieder, noch während sie die Deportation der bulgarischen Juden beschlossen, an ihn, um ihre persōnlichen jüdischen Freunde zu retten. Die Sofioter Juden baten in erster Linie Politiker der Opposition wie Mušanov, Stajnov, den Metropoliten Stefan, Dimo Kazasov und Damjan Velčev um Unterstützung.

43 Chary, Bulgarian Jews, S. 84-87.

44 Ebenda, S. $88 \mathrm{f}$. 
Vom Handelsminister Nikola Zachariev erhielt Baruh die wichtige Information, daß der Kabinettsbeschluß lediglich die Deportation der Juden aus den besetzten Gebieten und nicht der aus Altbulgarien erlaubte.

Entscheidend war Baruhs Kontakt zu Dimitǔr Pešev, dem Abgeordneten von Kjustendil und Vizeprāsidenten des Parlaments. Am Morgen des 7. Mārz hatte er ein lāngeres Gespräch mit ihm, in dem er die Diskrepanz zwischen dem Kabinettsbeschluß und der geplanten Aktion erwāhnte. Pešev rief sofort Mitenov und den Polizeichef von Kjustendil an, die beide Baruhs Aussagen bestātigten. Währenddessen setzte das Kommissariat seine Vorbereitungen fort. Als der zuständige KEV-Delegierte Tasev erfuhr, daß Gouverneur Mitenov bereits Samuel Baruh informiert hatte und die geplante Aktion nicht mehr geheim war, beschloß er, die Juden in Kjustendil schon einen Tag früher, am 9. März, zusammenzutreiben. Auch hatte er gehört, daß der Abgeordnete Petŭr Mihalev von der Aktion wußte und daß eine Delegation von fünf Personen von Kjustendil nach Sofia reisen wollte. Tatsāchlich konnten nur zwei Reprāsentanten den Zug nach Sofia besteigen, die anderen wurden vom Ratnici-Mob an der Abreise gehindert. Am Morgen des 9. Mārz trafen in Peševs Büro mehrere Juden und Bulgaren zusammen, die beschlossen, die Regierung kurz vor Beginn der Parlamentssitzung noch am selben Nachmittag mit ihrem Protest zu konfrontieren. Pešev bat am Nachmittag Innenminister Gabrovski in sein Büro, wo eine Gruppe von Abgeordneten auf ihn wartete. Sie teilten ihm mit, daß sie von den Deportationsplānen wüßten, auch über die aus Altbulgarien, die dem Kabinettsbeschluß widersprāchen. Nach Rücksprache mit Filov erklärte Gabrovski, der Beschluß könne nicht revidiert werden, jedoch stimme der Regierungschef einem Aufschub der Deportationen aus Altbulgarien zu. Wahrscheinlich hatte Filov zuvor König Boris konsultiert.) Am Abend forderte Pešev den Innenminister auf, die erlassenen Befehle zu widerrufen. (Die Juden sollten gegen Mitternacht interniert werden.) Pešev verlangte unverzügliches Handeln und rief schließlich Mitenov in Kjustendil selbst an und teilte ihm mit, daß Gabrovski die Aktion stoppen wolle. Andere Abgeordnete informierten ihre Bezirke. Schließlich befahl Gabrovski, um nicht die Initiative zu verlieren, seinem Sekretār, allen Stādten in Altbulgarien zu telegraphieren, daß die Befehle zur Arretierung der Juden aufgehoben seien. Die telegraphische Botschaft erreichte jedoch die zustāndigen Stellen erst am nāchsten Tag, am 10. Mārz. In Plovdiv, Pazardžik, Samokov, Haskovo, Sumen und in einigen anderen Stādten hatte die Polizei die Juden bereits in Schulen, Synagogen und Lagem zum Abtransport zusammengetrieben; als die neuen Instruktionen eintrafen, wurden die Juden jedoch sofort freigelassen.

Das Kommissariat blies die Aktion ab und rief seine Mitarbeiter zurück; das Scheitern der Aktion führte man auf die mangelnde Diskretion zurück. Pešev gab sich jedoch mit dem Aufschub der Aktion nicht zufrieden. Denn wāhrend das KEV die Deportation der Juden aus Thrazien und Mazedonien in jenen Wochen durchführte, bestand die Gefahr, daß auch die Aktionen gegen die bulgarischen Juden wieder aufgenommen wurden. Am 17. Mārz sandte Pešev einen von 42 Abgeordneten (hauptsāchlich aus der Regierungsfraktion) unterzeichneten Protestbrief an Ministerpräsident Filov, in dem die Judenpolitik der Regierung scharf verurteilt und als für das Ansehen und die Interessen der Nation schädlich bezeichnet wurde. Sie wiesen darauf hin, daß die Vertreibung der Juden aus dem Lande letztlich zu ihrem Tode führen würde - eine schwere Beschuldigung, da die „Endlösung “ ja nicht öffentlich propa- 
giert worden war. Zu den Unterzeichnern gehörten Prominente wie Andro Lulčev, der Bruder des kōniglichen Beraters, Dr. Petur Kjoseivanov, der Neffe des ehemaligen Premiers, Dr. Ivan Vazov, der Neffe des berühmten Schriftstellers, einige Abgeordnete aus Südwestbulgarien, Mušanov von der Opposition (Petko Stajnov unterzeichnete nicht, drückte aber seine Sympathie aus) und zur großen Uberraschung der rechte Oppositionsführer Alexander Cankov, Günstling der Deutschen, der allerdings mit seiner Unterschrift mehr die Regierung provozieren als den Juden nützen wollte.

Pešev übergab dem Premier den Protest am Morgen des 19. Mārz. Am Nachmittag führte ein anderer Abgeordneter eine Gruppe von Befürwortern der Regierungspolitik an, die dem Protest entgegentraten. Am nāchsten Tag sprach Filov mit Jordan Sevov, dem einflußreichsten Königsberater. Auf einer Kabinettssitzung beschloß man, den Protest zum Testfall für die Klārung der Regierungsmehrheit im Parlament zu nehmen. Auch wollte man die Unterzeichner aus der Regierungsfraktion unter Druck setzen. Einige Abgeordnete zogen ihre Unterschrift wieder zurück. Nach langer Debatte stimmte am 24. März die Mehrheit im Parlament für den Regierungskurs, einschließlich der Judenpolitik. Der Protest wurde zurückgezogen. Für den Antrag, Pešev zu rügen, stimmten nur 66 Abgeordnete. Das Verhalten vieler Parlamentarier war widersprüchlich, indem sie der Regierung ihr volles Vertrauen ausdrückten, sich aber weigerten, Pešev zu rügen. Am 26. Mārz wurde der Antrag gestellt, ihn von seinem Posten zu entfernen. In einer turbulenten Sitzung trat Pešev nach einer öffentlichen Erklärung zurück. In derselben Sitzung wurde auch ein Dekret über die Staatsbürgerschaft in den neuen Gebieten verabschiedet, das dem Zweck diente, die Griechen aus Mazedonien und Thrazien zu vertreiben. Petko Stajnov wies dabei auch auf die Bedeutung des Dekrets für die Juden hin, die bulgarische Bürger seien und nicht gewaltsam aus Bulgarien vertrieben werden dürften.

Beckerle versuchte in seinem Bericht über die spektakulāre Sitzung die Bedeutung des Protests herunterzuspielen und hob die Tatsache hervor, daß Pešev zurückgetreten sei und selbst die Mitglieder der Regierungsfraktion, die den Protest unterzeichnet hātten, den Maßnahmen zur Staatssicherheit zugestimmt hātten. Es schien, als sei der Protest ein Fehlschlag gewesen. Die Regierung hatte eine ausreichende Mehrheit, die obendrein diejenigen, die ihren Protest aufrechterhielten, noch bestrafte. Die Diskussion über die Judenpolitik wurde aus dem Parlament verwiesen. Zur gleichen Zeit liefen die Deportationen aus den besetzten Gebieten weiter und man erwartete, daß die Juden aus Altbulgarien als nāchste folgen würden.45

\section{Die Deportation der Juden aus Thrazien}

Die Deportation der thrazischen Juden begann am 4. März 1943, noch vor Peševs Protestaktion. In allen Städten Ost-Thraziens mit nennenswertem jüdischen Bevölkerungsanteil - Gjumjurdžina, Dede agač, Kavalla, Drama, Ksanti und Seres - gingen die KEV-Vertreter, entsprechend den Anweisungen von Belev und Jaroslav Kalicin, der die Deportation in dieser Region überwachte, in derselben Weise vor: Während der

4 Ebenda, S. 90-100; Bericht Beckerles vom 26. Mär 1943 (173863-864), in: PA, Büro Staatssekretär Bulgarien, Bd. 5. 
Dauer der Aktion, die manchmal nach Mitternacht begann und bis sieben oder acht Uhr morgens dauerte, stellte die Polizei die Stādte unter Blockade und verhāngte Ausgehverbot. Kurz bevor die Aktion gegen vier Uhr morgens begann, erhielten die Polizisten in Dreiergruppen ihre Instruktionen, einschließlich einer Liste der jüdischen Familien, die deportiert werden sollten und der notwendigen Ausrüstung zum Versiegeln der jüdischen Häuser. Die thrazischen Juden wußten wohl, daß etwas bevorstand, kannten aber nicht den genauen Zeitpunkt der Operation. Das Wecken mitten in der Nacht überraschte sie. Gemäß Kalicins Plan teilte die Polizei den Juden mit, daß die Regierung sie ins Landesinnere von Bulgarien verschicken wolle und daß sie bald wieder in ihre Heimat zurückkehren kōnnten. 46

Die Juden marschierten durch die Hauptstraßen der Städte, wobei ihre Zahl an jeder Kreuzung anschwoll, bis sie ihr Ziel erreichten - die Tabaklager, die vorübergehend als Sammellager dienten. Dort blieben sie ein bis zwei Tage, bis sie zu den gröBeren Deportationszentren in Dupnica und Gorna Džumaja geschickt wurden. 77 Das deutsche Generalkonsulat in Kavalla berichtete der deutschen Gesandtschaft in Sofia über die Deportation48: „Der Abtransport der Juden aus dem Belomoriegebiet ist ... zum größten Teil abgeschlossen. Ein Teil der Juden befindet sich mit Gepäck auf dem Wege nach dem Sammellager Gomadjoumaja, ein anderer Teil ist dort bereits eingetroffen und interniert. Nach bislang vorliegenden Meldungen sollen insgesamt etwa 4500 Juden im Belomoriegebiet erfaßt worden sein. Soweit ich ermitteln konnte, gestaltet sich ihre Abschiebung ohne besondere Schwierigkeiten und Zwischenfälle. Bemerkenswert war nur ... die offensichtliche Anteilnahme der griechischen Bevölkerung, die z.B. in Kavalla und Drama den abziehenden Juden Geschenke und sonstige widerlich-innige Abschiedsovationen darbrachte. Wie ... von zuverlässiger deutscher Seite mitgeteilt wurde, haben sich an dem unerfreulichen Schauspiel in Drama auch einige offenbar kommunistisch angehauchte Bulgaren beteiligt. Die Juden selbst sollen die Abschiebung wenigstens nach außen hin gleichgültig aufgenommen haben."

In dem Bericht sind 4500 Juden genannt. Nach einer KEV-Erhebung vom Jahre 1942 lebten in Thrazien (in fünf Kommissariatsbereichen) 3975 Juden - eine offensichtlich zu niedrig angesetzte Zahl.49 Nach einem griechischen Bericht waren es 19405490 Juden, und nach Angaben des KEV wurden 19434273 registriert $^{\text {50; zur }}$ Deportation gelangten aber nur 4058, d.h. mehr als $20 \%$ bzw. über 200 Juden entgingen der Deportation: Die meisten davon wurden nach Süden, nach Griechenland, ausgewiesen. Viele hatten fremde Staatsbürgerschaft; so schickten die Bulgaren Juden aus Lāndern, die von den Deutschen besetzt waren, in die Lager; die Staatsangehörigen neutraler und unabhängiger Achsen-Verbündeter wie Italien, Spanien und der Türkei entgingen meist der Deportation. Viele Juden blieben wegen Krankheit, wegen des Einsatzes in Arbeitstrupps oder aus anderen Gründen verschont. 51

Nach bulgarischen Angaben wurden aus folgenden Städten Thraziens Juden deportiert $\$ 2$ :

46 Chary, Bulgarian Jews, S.101 f.

47 Ebenda.

48 Bericht Drägers vom 9. März 1943 (486308), in: PA, Inland II B, Judenfrage in Bulgarien (1941-1944).

49 Chary, Bulgarian Jews, S. 104.

50 Ebend.

51 Ebenda.

52 Zehlenangaben laut Berichten des Kommissariats, kritisch kommentiert von Chary, Bulgarian Jews, S. 105. 


\begin{tabular}{lccc}
\hline Stadt & Personen & deportiert & nicht deportiert \\
\hline Dede agač & 44 & 42 & 2 \\
Drama & 592 & 589 & 3 \\
Gjumjurdžina & 904 & 878 & 26 \\
Kavalla & 1657 & 1484 & 173 \\
Ksanti & 537 & 526 & 11 \\
Pravište & 19 & 19 & 0 \\
Samotraki & 3 & 3 & 0 \\
Sarz-Saban & $11^{\circ}$ & $12^{\circ}$ & 0 \\
Seres & 471 & 471 & 0 \\
Tasos & 16 & 16 & 0 \\
Ziljachovo & 19 & 18 & 1 \\
\hline insgesamt & 4273 & 4058 & 216 \\
\hline
\end{tabular}

- Der Bericht des Kommissariats gibt keine Erklärung für diese Diskrepanz. Eventuell zusātzliche Person durch Zuzug oder Geburt in der Zeit zwischen der Registrierung der Personen und dem Zeitpunkt der Deportation.

Den Juden in Thrazien war es nicht erlaubt, die bulgarische Staatsbürgerschaft zu erwerben. Die meisten von ihnen behielten die griechische Staatsangehörigkeit. Als die Lage kritisch wurde, erwarben viele Familien die spanische Staatsbürgerschaft, wobei sie ihren Anspruch von ihren sephardischen Vorfahren herleiteten. Großzügig waren auch die Italiener bei der Verleihung ihrer Staatsbürgerschaft an die Juden. ${ }^{33}$ Viele ausländische Juden waren von der bulgarischen Aktion zunāchst betroffen, wurden dann aber wieder freigelassen. Juden bulgarischer Staatsangehörigkeit, die in Thrazien zum Arbeitsdienst mobilisiert waren, wurden ebenfalls verhaftet, doch nach den Ereignissen vom 9. März wieder entlassen. Einige Juden aus Thrazien waren in die Arbeitstrupps für Straßen- und Eisenbahnbau in Südwestbulgarien eingezogen. Ungefähr 42 von ihnen wurden deportiert. Andere in Arbeitslagern internierte thrazische Juden wurden zu spāt inhaftiert und entgingen so der Deportation.

Gemäß den Berichten der KEV-Vertreter wurde die thrazische Operation erfolgreich abgeschlossen. Sie erfolgte nach Plan, ohne größere Abweichungen. Verschwiegen wurden die Tragödien und das Leid, das sich bei den Aktionen abgespielt hatte: Die Juden wurden aus ihrer Heimat ohne angemessene Vorwarnung verschleppt, ohne ausreichende Nahrung, Wasser, sanitäre Anlagen und medizinische Versorgung in Lagern zusammengepfercht; sie wurden Entlausungsaktionen und erniedrigenden Untersuchungen unterzogen, wobei ihnen die wenige Habe, die ihnen die Polizei mitzunehmen erlaubt hatte, auch noch abgenommen oder beschädigt wurde. Die lange Reise in offenen Wagen durch Thrazien war beschwerlich. Viele wurden krank, einige starben. Manche Frauen brachten in diesem Elend Kinder zur Welt. Es herrschte unter den Deportierten große Verzweiflung. Oft wurden sie von den Wachmannschaften grob behandelt, es gab aber auch Beamte, die das Unrecht der KEV-Aktion empfanden und die Juden anständig behandelten. 54

33 Chary, Bulgarian Jews, S. $105 t$.

54 Ebends, S. $107 f$. 
In Demir-Hisar und Simitli stiegen die Juden von Karren um in Züge, von denen sie zu den Sammellagern in Südwestbulgarien - Gorna Džumaja und Dupnica - transportiert wurden. Uber 2500 Juden wurden in Gorna Džumaja und weniger als 1500 in Dupnica untergebracht. Offensichtlich wurden nur Juden aus Gjumjurdžina und Ksanti nach Dupnica und der Rest nach Gorna Džumaja transportiert. Die Zustānde in den provisorischen Lagern (Tabaklager, Schulen usw.) waren schlecht, die Verpflegung und die medizinische Versorgung, die Hygiene mangelhaft. 55 In den Zwischenstationen revidierten die Beamten die ursprüngliche Version der Begründung der Judendeportation. Sie behaupteten jetzt, die Regierung habe mit den Briten die Ausreise der Juden nach Palāstina vereinbart, sie würden von den Sammellagem zur Adria- und Schwarzmeerküste transportiert. Aber die Juden glaubten ihnen nicht. 56

Für den Transport von den Sammellagern zu den unter deutscher Herrschaft stehenden Gebieten waren die bulgarischen Eisenbahnen zustāndig und das KEV verantwortlich. Bereits am Tage des Abschlusses des Dannecker-Belev-Abkommens wurden die bulgarischen Staatsbahnen vom KEV über die bevorstehende Aktion informiert. Das Kommissariat und die Eisenbahn arbeiteten zusammen einen detaillierten Plan aus. 57 Der Transport der thrazischen Juden zum Donauhafen Lom erforderte zwei Zugfahrten. Die erste sollte am 18. März mit über 2000 Personen von Gorna Džumaja mit eineinhalbstündiger Unterbrechung in Sofia abgehen und am 19. Mārz gegen 3.38 Uhr morgens in Lom enden. Der zweite Zug mit 600 Juden sollte am 19. Mărz Gorna Džumaja verlassen, die Juden in Dupnica (1500 Personen) und in Sofia ca. 200 Personen aus Pirot aufnehmen und am 20. März in Lom ankommen. Die Passagiere reisten von Gorna Džumaja, Dupnica und Pirot ohne Fahrkarten. In Sofia stellten Bahnbeamte fest, wieviele Personen in jedem Zug welcher Altersgruppe angehörten, denn Personen unter 4 Jahren fuhren frei, zwischen 4 und 10 Jahren zum halben Preis und ab 10 Jahren zum Erwachsenentarif. Doch lösten sie für alle den Arbeitertarif, d.h. die Hälfte des regulären Fahrpreises. Die Juden reisten von Sofia nach Lom mit einer Gruppenfahrkarte, die ausgestellt auf die Namen der Gruppenführer, vom Polizeichef oder vom Chef der uniformierten Polizei unterzeichnet wurde. Von Lom über Wien nach Kattowitz fuhren die Juden auf Danneckers Verantwortung. Spāter stellten die Deutschen dem Kommissariat die Reisekosten, für die die Bulgaren verantwortlich waren, in Rechnung. ${ }^{38}$

Die bulgarische Polizei diente nicht nur wāhrend der Fahrt durch Bulgarien, sondern bis nach Kattowitz, in Zusammenarbeit mit den Deutschen als Zugbewachung. Das KEV mietete zur Abfahrt von Lom vier Schiffe; auch dafür wurde bulgarisches Wachpersonal, insgesamt 86 Beamte, bereitgestellt. 59 Der erste Zug von Gorna Džumaja ging am 18. Mārz mit 1985 Juden ab; der zweite am 19. Mărz befōrderte 692 Personen. Von Dupnica fuhren 1380 Personen ab. Insgesamt verließen also $4057 \mathrm{Ju}$ den, darunter 681 Kinder unter 10 Jahren, aus Thrazien die beiden Abreisezentren, die Juden aus Pirot nicht mitgezāhlt. Während der 14-tägigen Reise durch Thrazien kamen einige Personen hinzu, insbesondere einige neugeborene Kinder, andere Juden

5s Ebenda, S. $109 \mathrm{f}$

56 Ebende, S. 111.

57 Grinberg, Dokumenti, S. $110 \mathrm{f}$.

58 Chary, Bulgarian Jews, S. 117 f.

59 Grinberg, Dokumenti, S. 112-116. 
verstarben. Doch hatte sich die Gesamtzahl gegenüber der ursprünglichen Gruppe von 4058 Personen nur geringfügig verändert, nāmlich auf 4057 Personen. Hinzu kamen 158 Juden aus Pirot. ${ }^{60}$ Das erste Schiff, die „Kara G'orgi“, verließ Lom um 2 Uhr nachmittags am 20. Mārz; das zweite, die „Voivoda Masil ${ }^{4}$ fuhr am selben Abend los. Zwei Schiffe, die "Satumus" und „Car Dušan“, legten am nächsten Abend ab. Jedes Schiff beförderte zwischen 875 und 1100 Passagiere, insgesamt verließen 4219 Juden Lom. Bulgarisches Wachpersonal stand bereit, doch die ganze Operation wurde von deutschen Beamten überwacht. Während der Fahrt auf der "Car Dušan starben sieben Personen, auf der ${ }_{n}$ Saturnus " eine; die Deutschen entfernten die Toten in einigen Donauhäfen von den Schiffen. Die „Car Dušan“ brauchte für die Reise nach Wien ungefähr zehn Tage. Die „Satumus“ erreichte Wien bereits nach fünf Tagen.61 Die àgāischen Juden wurden von der nordbulgarischen Stadt Lom aus in Schiffe verladen und auf der Donau nach Wien transportiert; insgesamt wurden 4219 Juden über Lom und Wien ins besetzte Polen deportiert.62

\section{Judendeportationen aus dem Pirot-Distrikt}

Am brutalsten liefen die Deportationen in dem Gebiet um Pirot ab, einem kleinen Distrikt Ostserbiens - ca. $60 \mathrm{~km}$ nordwestlich von Sofia, der 1941 an Bulgarien gefallen war. Die technische Durchführung der Operation āhnelte der in Thrazien. Am 11. Mārz erhielten die Behörden den Befehl, mit der Aktion zu beginnen. Am nächsten Tag trieben Polizeipatrouillen die Juden aus ihren Hāusern, befahlen ihnen, ihre Wertsachen einzusammeln und abzugeben, gaben ihnen kaum eine halbe Stunde, ihre Sachen zu packen, und versiegelten die Häuser. Danach mußten die Juden zum Piroter Gymnasium marschieren, wo sie bis zu ihrem Abtransport interniert wurden. Dort durchsuchte man sie in erniedrigender Weise nach Wertsachen.

Von der Operation waren alle Juden der Stadt, auch die mit bulgarischer Staatsbürgerschaft, betroffen, die von Bulgarien aus in Pirot zum Arbeitsdienst eingesetzt worden waren. Insgesamt wurden 188 Personen in Pirot interniert. Davon waren 27 Juden bulgarischer Staatsbürgerschaft, die nicht deportiert wurden. Am Tage der Deportation, am 19. März 1943, verließen 158 Juden Pirot in Richtung Sofia, wo sie in die Züge steigen sollten, die die Juden aus Thrazien beförderten. An sich hätten $188 \mathrm{mi}$ nus 27 Personen, d.h. insgesamt 161, betroffen sein müssen, doch geben die Dokumente darüber keinen Aufschluß. Möglicherweise sind einige der älteren Juden vor dem Abtransport verstorben.63 Im September 1943 schickte die Regierung die übrigen Juden bulgarischer Staatsbürgerschaft in das Innere des Landes. Das Eigentum, das sie noch im März bei der Internierung behalten konnten, verloren sie zu diesem Zeitpunkt.

60 Chary, Bulgarian Jews, S. 118.

61 Grinberg, Dokumenti, S. $116 f$.

62 Beckerle an AA, 26. Mär 1943 (486314), in: PA, Inland II g, Judenfrage in Bulgarien (1941-1944).

63 Chary, Bulgarian Jews, S. 114-117. 


\section{Deportation der mazedonischen Juden}

Die Juden aus Mazedonien waren die letzte Gruppe, die von den Bulgaren deportiert wurde. Die Aktion begann eine Woche nach der in Thrazien und auch nach den abgebrochenen Vorbereitungen für die Umsiedlung der bulgarischen Juden ins Landesinnere. Viele mazedonische Juden waren durch die Maßnahmen in den Nachbargebieten schon vorgewarnt. Uber 100 Juden gelang es, rechtzeitig vor Beginn der Aktion über die Grenzen in das von den Italienem besetzte Gebiet Albaniens zu entkommen. ${ }^{64}$ Um weitere Flucht zu verhindern, verstäkten die Bulgaren ihre Bewachung an der albanischen Grenze. Ursprünglich sollten die mazedonischen Juden in den beiden größten Städten Skopje und Bitola, in denen jeweils über 3000 Juden lebten, interniert werden. Doch hatte Bitola keinen geeigneten Platz, wo man die Juden hätte unterbringen können. So wurden sie zum Abtransport alle in Skopje in den Gebāuden des staatlichen Tabak-Unternehmens gesammelt. Der bulgarische Judenkommissar Belev kam eigens nach Mazedonien, um die Aktion zu überwachen, denn es war die größte Einzelaktion dieser Art.

Nach Angaben des Kommissariats (vom Mārz 1943) lebten vor der Aktion in Mazedonien in Bitola 3342 Juden, in Skopje 3493 und in Stip 546, insgesamt 7381 Personen.65 Nach Statistiken jugoslawischen Ursprungs aus der Zeit vor dem Kriege liegen für die jüdische Minorität in Mazedonien folgende Zahlen vor66:

\begin{tabular}{lr}
\hline Stadt & Personen \\
Bitola & 3351 \\
Stip & 551 \\
Skopje & 3795 \\
Gevgelija & 11 \\
Kriva Palanka & 5 \\
Kumanovo & 13 \\
Veles & 8 \\
sonstige & 28 \\
\hline insgesamt & 7762
\end{tabular}

Unmittelbar nach dem Balkanfeldzug nahm die Zahl infolge der Emigration aus Serbien leicht zu, aber offenbar verließen über 500 Juden Mazedonien zwischen April 1941 und September 1942.

Die Maßnahmen verliefen nach der gleichen Art wie in Thrazien und Pirot. Die Aktion begann am 11. März. Die Ortspolizei verhaftete die Juden in Bitola und Stip und transportierte sie per Bahn nach Skopje. Den im Tabaklager in Skopje internierten Juden wurde wie in den anderen Gebieten mitgeteilt, sie würden ins Landesinnere Bulgariens transportiert, doch konnten die Mazedonier diese Lüge kaum glauben, da sich das Schicksal der Juden jenseits der Grenze schon längst herumgesprochen hatte. Nach Angaben des KEV-Vertreters in Skopje Peju Draganov Peev wurden am

64 Grinberg, Dokumenti, S. 81.

65 Chary, Bulgarian Jews, S. 122.

66 Elaborat za zlodelata na okupatorite i nivnite pomagateli nad evrejskoto naselenie vo Makedonija, 2. April 1946, S. 1; Aleksander Matkovsky, The Destruction of Macedonian Jewry, in: Yad Vashem Studies on the European Jewish Catastrophe and Resistance 3 (1959), S. 207 f.; Chary, Bulgarian Jews, S. 123. 
11. Mārz 7215 Juden ins Lager eingeliefert, davon 3313 aus Skopje, 3351 aus Bitola und 551 aus Stip. 25 Personen kamen kurz darauf. Von den Lagerinsassen waren 2300 unter 16 Jahre alt, darunter 1100 unter 10.67 Aleksander Matkovsky kommt in seiner Monographie über die Deportation der mazedonischen Juden auf über 7300 Juden, die ins Lager Skopje eingeliefert wurden, einschließlich derjenigen, die von den Behörden später wieder freigelassen wurden oder entkommen konnten.

Die Bulgaren entließen 77 Ärzte und Apotheker mit ihren Familien wegen des groBen Mangels in dieser Berufssparte. Auch Juden auslāndischer Staatsbürgerschaft wurden freigelassen, und zwar 74 Spaniolen, 19 Albaner, 5 Italiener, doch 12 Personen dieser Gruppe wurden von den Deutschen spāter wieder verhaftet. 68 Zwei weitere entkamen, so daß insgesamt 155 Juden von den Skopjer Lagerinsassen der Endlōsung entgingen. Vier Juden starben im Lager vor dem Abtransport. Einige Insassen des Lagers Skopje (47 nach Angaben Grinbergs) kamen aus Thrazien und Pirot. Eine gewisse Anzahl der 7381 jüdischen Bewohner Mazedoniens (nach dem Zensus des Kommissariats) entging der Einlieferung ins Lager; die meisten konnten über die albanische Grenze vor der Aktion fliehen.

Die Lagerinsassen aurden in drei Transporten nach Polen weitergeleitet - am 22., 25. und 29. März. Von Skopje ging die Fahrt über Niš, Gurlica (italienisches Besatzungsgebiet), Lapovo (deutsche Zone) durch Albanien, Serbien, Kroatien, Ungarn, Deutschland nach Treblinka im Generalgouvernement. Die bulgarischen Staatsbahnen arrangierten den Transport; die Fahrkarten für die Juden löste das Kommissariat beim bulgarischen Reisebüro Balkan. In Lapovo übernahmen deutsche Stellen den Transport von den Bulgaren. Wie bei den anderen Deportationen bestand die bulgarische Bewachung aus 43 Beamten pro Zug.

Nach Matkovsky beförderte der erste Transport 2338 Juden, der zweite 2402, der dritte 2404 - insgesamt also 7144 Personen. Zwölf Personen starben unterwegs. Diese Angaben beruhen auf deutschen Dokumenten, die wahrscheinlich die genauesten sind. Kleinere Abweichungen ergeben die Zahlen des Kommissariats: Grinberg zählt in den drei Transporten 2409, 2399 und 2315 Personen, zu denen noch 42 aus Thrazien beim letzten Transport hinzukommen, was insgesamt 7165 Personen ergibt. Draganovs Angaben, wiedergegeben von Matkovsky, bringen eine dritte Gesamtzahl, nämlich 7069 , die jedoch zu niedrig angesetzt ist. 196 mazedonische Juden kehrten nach dem Kriege nach Jugoslawien zurück - 116 von Albanien, 15 aus deutschen Lagern und 65 aus verschiedenen Konzentrationslagern. Wahrscheinlich gehörte nur die letzte Gruppe zu den ursprünglich Deportierten. 69

Die deutsche Berichterstattung in Berlin gibt als Gesamtzahl 7240 an, was ungenau ist; wahrscheinlich ist dies die Zahl der ursprünglich im Skopjer Lager Internierten. Polizeiattaché Hoffmann berichtete, daß 7122 mazedonische Juden aus Skopje abtransportiert wurden - diese Zahl kommt den anderen Angaben näher und ist auch rechtlich akzeptiert. ${ }^{70}$

67 Chary, Bulgarian Jews, S. $123 \mathrm{f}$

68 Ebenda, S. 124.

69 Ebenda, S. $124 \mathrm{f}$.

70 Zur Deportation der mazedonischen Juden siehe den Bericht des Deutschen Generalkonsuls in Skopje, Witte, 18. März 1943, an die Deutsche Gesandtschaft in Sofia (486309-315) sowie die Berichte von Wagner vom 3. April 1943 und Hoffmann vom 5. April 1943 (486326-330, 486316-321), in: PA, Inland II g, Judenfrage in Bulgarien (1941-1944). 


\section{Gesamtzahlen zur Deportation aus den bulgarisch besetzten Gebieten}

Nach Schātzungen aus den verläßlichsten Statistiken deportierten die Bulgaren 4057 Juden aus Thrazien (Chary gibt die Zahl der thrazischen Juden mit 4075 an [Druckfehler?], woraus sich die weiteren Zahlen ergeben. Die Zahl 4075 weicht von den vorhergenannten Zahlen 4057 [4058] völlig ab; vgl. Statistik thrazische Juden), 158 Juden aus Pirot und 7160 Juden aus Mazedonien - insgesamt also 11393 Personen. Diese Ziffern basieren aut deutschen und bulgarischen Transportzahlen, korrigiert durch Angaben von Todesfällen in den Sammellagern. In den Zahlen sind auch Juden aus anderen Gebieten enthalten, so sind thrazische Juden in der Ziffer für Mazedonien enthalten. Nach Angaben des Kommissariats lebten vor der Deportation 4273 Juden in Thrazien, 186 in Pirot und 7381 in Mazedonien, insgesamt also 11840 Juden im gesamten Okkupationsgebiet. Nach diesen Zahlen gelang es 465 Juden, der Deportation zu entkommen. Die meisten von ihnen hatten fremde Staatsbürgerschaft. Einige flohen, bevor die Aktion begann, oder konnten sich während ihres Ablaufs versteckt halten. ${ }^{71}$

Deutsche Berichte in Berlin geben eine Gesamtzahl von 4219 Juden aus Thrazien einschließlich Pirot und 7240 aus Mazedonien, insgesamt also 11459 Deportierte an. Andere deutsche Berichte zāhlen 4221 Juden aus Lom und 7122 aus Skopje, also insgesamt 11343 Deportierte. Die letzteren Transportzahlen aus dem Bericht des Polizeiattachés Hoffmann sind auch den Nachkriegsprozessen zugrundegelegt, wie z.B. im Frankfurter Prozeß gegen den deutschen Gesandten in Sofia, Adolph-Heinz Beckerle. Schließlich ist noch in einer Auflistung des Statistikbeauftragten Korherr an Himmler die Gesamtzahl 11364 angegeben. ${ }^{72}$

Eine Bestātigung der in den Prozessen akzeptierten Ziffer ergibt sich aus der Berechnung der Deportationskosten: So forderten die Deutschen außer den Fahrkosten für jeden Deportierten 250.- RM. Auf der Basis von Hoffmanns Angaben von 11343 Deportierten verlangten sie von den Bulgaren 2835750 . - RM. Die Bulgaren hielten diesen Preis für zu hoch; man kam in dieser Frage zu keiner Ubereinkunft und es wurde auch nichts gezahlt. ${ }^{73}$

\section{Rettungsaktionen zugunsten der bulgarischen und ausländischen Juden in Bulgarien}

In Bulgarien ging es nicht nur um die Rettung der einheimischen Juden, sondern auch um die aus anderen Lāndern, denn Bulgarien war aufgrund seiner Lage Durchgangsstation für Tausende von auslāndischen Juden. Das Schicksal der Juden im bul-

71 Chary, Bulgarian Jews, S. 127.

72 Berichte Hoffmanns vom 5. April 1943 und Kortherrs vom 19. April 1944; siehe auch Chary, Bulgarian Jews, S. 127. Zum Verfahren gegen den deutschen Gesandten Beckerle siehe insbesondere die Anklageschrift der Staatsanwaltschaft Frankfurt/Main (gegen Beckerle und von Hahn) vom 23. Dezember 1965 sowie das Urteil des Landgerichts Frankfurt/Main vom 19. August 1968 (gegen von Hahn): Aktenzeichen Js $2 / 63$ (Ks 2/67) (GStA) = V 502 AR-Z 59/59 - SA 324 - Zentrale Stelle Ludwigsburg.

73 Chary, Bulgarian Jews, S. 128. 
garischen Raum erregte auch die Weltöffentlichkeit. Am 11. März 1943, als die Aktionen gegen die mazedonischen und thrazischen Juden anliefen, erschien der schweizerische Gesandte Charles Redard als Vertreter des Internationalen Roten Kreuzes bei Ministerpräsident Filov und bot an, sich bei den Briten für ihre Aufnahme in Palāstina einzusetzen. Filov antwortete, es sei schon zu spät, in wenigen Tagen würden sie nach Polen deportiert. „Das heißt, in den sicheren Tod! meinte der Gesandte. Filov protestierte gegen diese Behauptung und erklārte, sie würden in Polen arbeiten wie die Bulgaren in Deutschland. In einer Unterredung wenige Tage später soll König Boris den Regierungschef gebeten haben, in der Judenfrage ${ }_{n} \mathrm{fest}^{\mathrm{a}} \mathrm{zu}$ bleiben. Wie auch in anderen kritischen Situationen überließ er die Entscheidung nachgeordneten Stellen.74 Ende Mai intervenierten zwei Vertreter des Internationalen Roten Kreuzes, Dr. Edouard Chapuissa und David de Trotz, beim König, beim Metropoliten und anderen Stellen wegen der bulgarischen Juden. König Boris sicherte zu, daß sie nicht deportiert und daß die Juden aus Sofia lediglich aufs Land umgesiedelt würden. Das Treffen fand zu einem Zeitpunkt statt, als die bulgarische Führung nach der Pešev-Aktion ihre Haltung in der Judenfrage radikal geändert und die Deportationsplāne zumindest aufgeschoben hatte.75

Im Herbst 1942 hatten bulgarische Juden in New York und in Palāstina ein Komitee zur Rettung der Juden in Bulgarien gegründet, das mit zionistischen Organisationen zusammenarbeitete und von Jaques Asseov, einem reichen Tabakhändler geleitet wurde. Sein Ziel war bescheiden - zunächst die Aussiedlung von 500 bulgarischen Kindern nach Palāstina zu erwirken.76 Die Initiativen des State Departments von 1942/43 verliefen im Sande; die Briten sahen durch eine Ausreise von Juden aus Europa ihre Palāstinaplāne gestōrt. Immerhin stimmten sie der Ubersiedlung von 500 jüdischen Kindern zu. Für $\mathbf{4 0 0 0}$ rumānische, ungarische oder bulgarische Kinder sowie für 500 Erwachsene war die Erteilung von Visa angebahnt. 77 Die schweizerische Gesandtschaft wandte sich Anfang Februar 1943 wegen der Abreise der jüdischen Kinder an die bulgarische Regierung. Da Filov im Hinblick auf die öffentliche Meinung und aus Furcht vor alliierten Repressalien die Briten nicht brüskieren wollte, schob er "technische Gründe“ vor und teilte der Schweiz mit, Bulgarien kōnne zur Zeit keine Transportzüge freistellen, denn sie würden für weitaus größere Transporte in der umgekehrten Richtung benōtigt; außerdem wolle man nicht die Kinder von ihren Familien trennen. ${ }^{78}$ Als die Sofioter Zeitung "Zora" weiter über Ausreisepläne berichtete, glaubten die deutschen Stellen an ein geheimes Arrangement zwischen Bulgaren und Briten in dieser Frage. Die bulgarische Regierung dementierte. 79 Dann tauchten Meldungen auf, 150 jüdischen Kindern aus Rumānien sei die Durchreise durch Bulgarien erlaubt worden. Das Reichssicherheitshauptamt verlangte, daß Sofia die Durchreise

74 Filov-Tagebuch, Eintragungen vom 11. und 15. Män 1943, in: Naroden süd, Nr. 3, S. 2; Bericht Beckerles vom 12. Mär 1943 (K 207336 in: PA, Inland II g, Ausreise der Juden nach Palastina.

75 Bericht Hoffmanns vom 24. Juni 1943 (173953-956, in: PA, Büro Stantssekretär Bulgarien, Bd. 5.

76 Chary, Bulgarian Jews, S. 131.

77 Siehe die Berichte im Aktenband Inland II g, Ausreise der Juden nach Palästina.

78 Berichte Beckerles vom 16. und 27. Februar 1943 (K 207372, K 207370-371), in: PA, Inland II g. Ausreise der Juden nach Palāstina. Bericht Hoffmanns vom 5. April 1943 (586316-321), in: PA, Inland II \&. Judenfrage in Bulgarien (1941-1944).

79 Pressebericht vom 2. März 1943 (K 207360); Rademacher an Deutsche Gesandtschaft Sofia, 2. März 1943 (K 207361); Beckerle an AA, 4. Mārz 1943 (K 207342), in: PA, Inland II g, Ausreise der Juden aus Palästina. 
verhindere. ${ }^{80}$ Tatsāchlich war nur vereinzelt Juden die Ausreise aus Bulgarien gestattet worden. 81

In der Frage der jüdischen Kinder aus Rumānien versicherte Filov Ende März, die Bulgaren versuchten alles, um den Transit von Juden zu stoppen, doch könnten sie nicht verhindern, daß einige durchkämen.82 Im Auswärtigen Amt war man mit den Bulgaren unzufrieden, die zwar zusicherten, die jüdische Emigration verhindem zu wollen, sie tatsāchlich aber weiter zuließen. So vermutete Staatssekretār Horst Wagner, das schwedische Schiff Öresund transportiere bulgarische Juden nach Palāstina. Bekkerle dementierte. ${ }^{83}$ Am 4 . April berichtete Killinger, 74 jüdische Kinder seien über Bulgarien auf dem Weg nach Palästina. Wagner forderte, Sofia solle die von der bulgarischen Gesandtschaft in Bukarest erteilten Visa widerrufen. Der Generalsekretār im bulgarischen Außenministerium Dimitǔr Sišmanov versicherte, die Visaerteilung werde aufgehoben und die Durchreise verhindert ${ }^{84}$

Im Mārz 1943 kam das Schicksal der Juden auf einem britisch-amerikanischen Gipfeltreffen in Washington zur Sprache, bei dem Roosevelt, der britische Außenminister Anthony Eden, sein amerikanischer Kollege Cordell Hull, Unterstaatssekretär Sumner Welles, der britische Botschafter in Washington Halifax, der Präsidentenberater Harry Hopkins und der stellvertretende britische Unterstaatssekretär William Strang zugegen waren. US-Außenminister Cordell Hull sprach die Frage der bulgarischen Juden an und erkundigte sich beim britischen Außenminister, ob für ihre Ausreise nach $\mathrm{Pa}$ lāstina gesorgt werden könne. Eden äußerte sich skeptisch, da die Rettung der bulgarischen Juden ähnliche Forderungen aus anderen Ländern nach sich ziehen könnte. Außerdem würde die Emigration einer großen Anzahl von Juden Transportprobleme aufwerfen. Die Briten seien aber bereit, 50000 Juden in Palāstina aufzunehmen, doch warnte er vor voreiligen öffentlichen Erklärungen. ${ }^{85}$

Am 25. März 1943 suchte ein bulgarischer Unterhāndler in Istanbul den amerikanischen Konsul Burton Y. Berry auf und berichtete vom Protest der Parlamentarier, der zum Aufschub der Deportationsplāne der bulgarischen Juden geführt habe. Er meinte, angesichts des wachsenden Prestiges der USA in Bulgarien könnte ein scharfer Protest der Amerikaner über die Schweizer Gesandtschaft in Sofia weitere Aktionen gegen die Juden verhindern und die Gegner der Judenpolitik im bulgarischen Kabinett stārken. Auch der ehemalige Präsident der bulgarischen landwirtschaftlichen Genossenschaftsbank und ein bulgarischer Anwalt sondierten in Istanbul wegen der Juden. Sie versicherten, daß nur 5 Prozent der Bulgaren das Bündnis mit der Achse und die Judenpolitik unterstützten. Auch die bulgarische Kirche und die Sofioter Universitāt seien gegen die Judenpolitik der Regierung. US-Außenminister Cordell Hull schickte über die Schweiz einen Brief an Filov mit der Aufforderung, die Juden in ein neutrales Land auswandern zu lassen. Die Schweizer leiteten die Note jedoch nicht weiter. Das

80 Siehe Aktenband Inland II g, Feldscher Aktion (Austausch jüdischer Kinder).

81 Killinger an AA, 24. Mär und 30. April 1943 (E 548802-803, E 421083-085); Wagner an Beckerie, 14. April 1943 (K 207316).

82 Chary, Bulgarian Jews, S. 134.

83 Bericht Wagners vom 3. Mär 1943 (486323), in: PA, Inland II g, Judenfrage in Bulgarien (1941-1944); Bekkerte an AA, 14. April 1943 (K 206942), in: PA, Inland II g, Juden, allgemein.

84 Chary, Bulgarian Jews, S. 135.

85 Siehe ebende, S. 135; Robert E. Sherwood, Roosevelt and Hopkins. An intimate History, New York 1948, S. 717; Hilberg, Destruction, S. 720 f. 
State Department übermittelte die Note schließlich dem bulgarischen Gesandten in Bern, Georgi Kjoseivanov. Der Expremier beantwortete die US-Note mit der Versicherung, Judenkommissar Belev sei bereits zum Rücktritt gezwungen worden, was allerdings nicht zutraf. ${ }^{86}$

Die Türkei beobachtete die anglo-amerikanischen Diskussionen über die Aussiedlung von Juden nach Palästina aufmerksam, denn sie bildete eine $Z$ wischenstation auf dem Weg dorthin. Der US-Botschafter in der Türkei, Laurence A. Steinhardt, selbst Jude, veranlaßte die Briten zum gemeinsamen Protest in Sofia, verbunden mit der Forderung, die Ausreise von 30000 bulgarischen Juden zu gestatten. Die Türken versprachen, die Durchreise der Juden zu erlauben, lehnten jedoch weitere Hilfsmaßnahmen wie die Einrichtung von Durchgangslagern unter verschiedenen Vorwānden ab. Die Alliierten erlangten zwar die Zustimmung des türkischen Außenministers, einigen Juden einen kurzen Aufenthalt in der Türkei zu gestatten, doch glaubte man nicht an einen Erfolg der Aktion. ${ }^{87}$ Auf amerikanischen und britischen Wunsch wandte sich die Türkei mit der Bitte an Bukarest, zwei rumānische Schiffe, die Transsylvania und die Bessarabia, für den Transport von Juden nach Istanbul bereitzustellen. Doch lehnten die Rumānen dies auf deutschen Druck hin ab.88 Auch die Bulgaren behandelten ein Ersuchen des Roten Kreuzes um ein Schiff oder um Transiterlaubnis für jüdische Emigranten abschlägig. So scheiterten die Plāne. ${ }^{89}$ Im Mai tauchten Gerüchte auf, die Schweiz habe mit Sofia über die Erteilung von 1500 Transitvisa verhandelt. Nach anderen Meldungen sollen die Bulgaren über den Austausch von 8000 bulgarischen Juden gegen 8000 Bulgaren aus Südamerika verhandelt haben. Doch Generalsekretär Sišmanov dementierte diese Meldungen und Beckerle unterstützte ihn darin. 90

Im Sommer 1943 wurden vom Westen erneut halbherzige Versuche unternommen, Juden zu retten. Uber die Schweiz forderte die britische Regierung Berlin auf, die Auswanderung von 5000 jüdischen Kindern aus Polen und anderen besetzten Gebieten nach Palästina zuzulassen. Prominente aus alliierten und neutralen Staaten setzten sich dabei besonders für die bulgarischen Juden ein. Das Rote Kreuz bat auBerdem die deutsche Botschaft in Ankara, 1000 bulgarische Juden per Schiff nach Haifa fahren zu lassen. Die Deutschen lehnten die britische Forderung nach jüdischer Emigration nach Palāstina mit dem Hinweis auf die Ungerechtigkeit gegenüber den Arabem ab, boten aber die Entsendung von Juden nach Großbritannien im Austausch gegen deutsche Kriegsgefangene an. Die Briten lehnten diesen Plan mit der Begründung ab, sie wollten deutsche Kriegsgefangene nicht gegen Personen austauschen, die nicht Bürger des Britischen Empire seien. Vom rumänischen Staatsführer Marschall Antonescu ging der Vorschlag aus, die Juden gegen angemessene Zahlungen austauschen zu lassen, auch dies wurde von westlicher Seite abgelehnt. Doch wuchs in den alliierten Ländern der Druck der Offentlichkeit, sich für die Juden einzusetzen. Neue Versuche erfolgten im Herbst 1943 und das Jahr 1944 hindurch.91

86 Chary, Bulgarian Jews, S. 136.

87 Ebenda, S. 137

88 Siehe Schriftwechsel im Maj 1943, in: PA, Inlend II G. Judenfrage in Bulgarien (1941-1944), und Inland II g, Feldscher Aktion.

89 Siehe Bericht des Geschäftsträgers in Sofia, Mohrmann vom 14. Mai 1943 (K 207441)

90 Beckerie an AA, 31. Mai 1943 (K 207427), in: PA, Inland II g, Ausreise der Juden nach Palastina.

91 Chary, Bulgarian Jews, S. $156 \mathrm{f}$. 
Im Oktober 1943 forderte die Schweiz im Auftrage der Briten die bulgarische Regierung auf, 5000 Juden nach Palāstina ausreisen zu lassen. Deutschem Druck ausgesetzt, keine Emigration zuzulassen, griffen die Bulgaren erneut zur Taktik - wie schon im Frühjahr 1943 -, die Ausreise aus technischen Gründen abzulehnen, wobei sie vermieden, die Alliierten zu brüskieren. Als Resultat der Verhandlungen kam es zu keinem Massenexodus aus Bulgarien. Ausreisevisa waren leicht zu bekommen, schwieriger war es, britische Einreisevisa nach Palāstina zu erhalten. Paradoxerweise wollte London die Masseneinwanderung nach Palāstina erlauben, die Einreise von Einzelpersonen hingegen erschweren. Der Strom jüdischer Migranten, der auch im Frühjahr 1943 nicht abgebrochen war, stieg gegen Ende des Jahres 194392 an. Der Großmufti von Jerusalem, Amin el Husseini, sandte 1943 über die Italiener mehrere Schreiben an Ribbentrop mit der Forderung, eine jüdische Emigration vom Balkan nach Palāstina nicht zuzulassen. Die halbherzigen Bemühungen der Alliierten um die Rettung der Juden auf dem Balkan hatten nur geringen Effekt. Erst angesichts ihrer militärschen Erfolge zeigte alliierter Druck in der Judenfrage in Sofia eine gewisse Wirkung. Aber solange Bulgarien weiterhin mit Deutschland verbündet blieb, mußte die Regierung in Sofia versuchen, in der Judenfrage weder die Deutschen noch die Alliierten zu verärgern. 93

\section{Die bulgarische Judenpolitik 1943/44}

Die bulgarisch-orthodoxe Kirche opponierte schon seit 1940 gegen die Judenpolitik der Regierung. Auch im Frühjahr 1943 setzten sich die Spitzen des Episkopats - der Metropolit von Sofia Stefan, die Bischöfe Kiril von Plovdiv und Neofit von Vidin - auf einer Audienz am 15. April 1943 bei König Boris, an der auch Ministerpräsident Filov teilnahm, für die Juden ein. Einigkeit bestand in der Schonung der Juden, die zum Christentum konvertiert waren. Schon vorher hatten Neofit und Stefan Zirkulare verbreitet, in denen sie den Kōnig wegen der Judenmaßnahmen verurteilten. In dieser Zeit bediente sich die Regierung einer massiven antisemitischen Propaganda, die wohl weniger die tatsāchliche Haltung der Regierung und des Königs ausdrückte, sondern der Rechtfertigung der Deportationen diente. 94

In der Krisensituation des Frühjahrs 1943 nach der Niederlage bei Stalingrad und in Erwartung einer alliierten Invasion auf dem Balkan, als die Partisanenaktivitāten zunahmen und Straßengefechte in der Hauptstadt tobten, erfolgte zwischen Februar und Mai 1943 eine Serie von Attentaten: So ermordete die berühmte jüdische Partisanin Violeta Jakova den Ex-Kriegsminister und Führer der rechten Legionāre General Lukov - ein Vorfall, den der König türkischen Agenten, Filov den Kommunisten zuschrieb und den Innenminister Gabrovski zum Anlaß einer antisemitischen Pressekampagne nahm.95 Am 15. April erschoß ein anderer Partisan den Abgeordneten

92 Ebenda

93 Ebenda, S. 138.

94 Ebend2, S. $138 \mathrm{f}$.

95 Mitka Grúbčeva, V imeto na naroda, Sofija, Izdatelstvo na BKP, 1962, S. 187-194; Filov-Tagebuch, Eintragungen vom 17. Februar und 15. April 1943, in: Naroden sùd, Nr. 3, S. 2 f. Bericht Beckerles vom 14. Februar 1943 (173816-817), in: PA, Büro, Staatssekretär Bulgarien, Bd. S. 
Sotir Janev; im Mai fiel der ehemalige Polizeichef Oberst Atanas Pantev Kugeln zum Opfer. Am spektakulärsten war der Anschlag auf Kǔnčo Janakiev, einen deutschen Agenten, denn der Täter wurde diesmal von der Polizei gefaßt - es war der Jude Menahem Papo, Mitglied des Arbeiterjugendverbandes und einer Partisanengruppe: Im nachfolgenden Prozeß wurde er wie 1941 Leon Tadžer zum Märtyrer, für die Regierung zur Symbolfigur ihrer Feinde.96

Trotz der von der Regierung Filov eingeschlagenen Linie mißtrauten die deutschen Stellen den Plānen der Bulgaren. Zwar äußerten sie ihre Zufriedenheit über die Judendeportationen aus den besetzten Gebieten, über den Stop der Deportationsplāne in Bulgarien selbst war man in Berlin aber enttāuscht: im März 1943 war die im Abkommen vereinbarte Zahl deportierter Juden nur zu 56 Prozent erreicht, statt der geplanten 20000 Juden waren nur ca. 11500 Juden abtransportiert worden. Die rechtsradikalen Legionäre übernahmen die deutsche Kritik und verurteilten in Pamphleten die Regierungspolitik. 97

Bei einem Besuch in Berlin im April 1943 betonte Zar Boris gegenüber Ribbentrop, daß gemäß dem Abkommen zwischen Dannecker und Belev nur die Juden aus den besetzten Gebieten deportiert werden sollten, was ja jetzt erfolgt sei. Auf dessen Hinweis, daß dies nur ein Vorspiel für die Deportation aller Juden aus Bulgarien sein könne, gestand Boris lediglich zu, daß „kommunistische Elemente“ unter den Juden deportiert werden sollten, während er den Rest von 25000 Juden in Bulgarien für öffentliche Arbeiten benötige. Boris plante offenbar, den Begriff ${ }_{n}$ kommunistisch“ sehr weit auszulegen. Ribbentrop gab sich damit nicht zufrieden und betonte die Notwendigkeit einer radikalen Lösung. 98

Innenminister Gabrovski hatte die Deportationsvorbereitungen in Bulgarien zwar auf Wink des Thrones aufgeschoben, aber wohl noch nicht endgültig aufgehoben. So entwarf Judenkommissar Belev einen stufenweisen Deportationsplan, von dem alle Juden in Bulgarien, ausgenommen die mit fremder Staatsangehörigkeit, die mit Christen verheirateten, die in Arbeitslagern mobilisierten sowie Schwerkranke, erfaßt werden sollten. Von den 25000 Juden in Sofia und 23000 in der Provinz sollten bis zum September 1943 monatlich 16000 auf Donauschiffen von Lom über Wien nach Polen verfrachtet werden. Erster Schritt im Gesamtplan war die Aussiedlung von ca. 16000 Sofioter Juden in verschiedene Provinzen (in die Region Burgas 3000, Pleven 1500, Plovdiv 1500, Ruse 2000, Sumen 1500, Sofia 2000, Stara Zagora 1500, Vraca 3000). Belevs Plan sah in der Version A die Deportation der Juden nach Polen aus Gründen der Staatssicherheit, nach Version B aber lediglich Umsiedlung in die Provinz vor. Am 20. Mai brachte Gabrovski den Plan dem König zur Kenntnis, der der Version B, nicht aber der Variante A zustimmte, was allerdings eine spätere radikale Lösung nicht ausschloß.99

96 Chary, Bulgarian Jews, S. 139 f.

97 Berichte Wittes vom 18. Mär 1943 (486309-313), Beckerles vom 21. März 1943 (486314); Bosshammer (RSHA) an Thadden (AA), 17. Maj 1943 (486341-343), in: PA, Inland II g. Judenfrage in Bulgarien (1941-1944).

98 Ribbentrop an Beckerle, 4. April 1943 (173890-891), in: PA, Büro Steatssekretār Bulgarien, Bd. S, Bosshammer an Thadden, 17. Mai 1943.

99 Dazu Belevs Bericht, abgedruckt in: Grinberg, Dokumenti, S. $185 \mathrm{ff}$; Bericht Hoffmanns an RSHA, 7. Juni 1943, in: PA, Inland II g. Judenfrage in Bulgarien (1941-1944). 
Am 21. Mai 1943 erhielten die Sofioter Juden die Aufforderung, die Stadt in drei Tagen zu verlassen und in die Provinz zu gehen, was Panik hervorrief - besonders im Ghetto „Juč Bunar “. Die Spitzen des Konsistoriums (Buko Levi) nahmen Fühlung mit einflußreichen Personen auf, wie dem Mazedonier-Führer Dr. Konstantin Stanišev, dem Königsvertrauten Dr. Handžiev, und der „Mutter des Hofes“ Jekaterina Karavelova, die versprach, beim Kōnig zu intervenieren. Auch Metropolit Stefan sagte einen Protest des Heiligen Synods gegen die neuerlichen Deportationsplāne zu. 100 Die Deportationen waren ausgerechnet auf den 24. Mai festgesetzt, dem St. Kyrill - und Methodius-Tag, der in Sofia mit Paraden und Gottesdiensten feierlich begangen wurde. Wie bei anderen kritischen Situationen hatte der König Sofia verlassen. Der Metropolit wurde bei der Regierung vorstellig und bezeichnete öffentlich die Plāne als Sünde und Entweihung des Feiertages. ${ }^{101}$

Unterstützung erhielten die Juden auch von der „Vaterländischen Front ${ }^{\text {, }}$, die die Juden im Ghetto „Juč Bunar ${ }^{\star}$ zu Demonstrationen und zur Selbstverteidigung aufrief. Vor allem Jugendliche beteiligten sich an Demonstrationen im Ghetto, gegen die Polizei und KEV-Beamte gewaltsam vorgingen. ${ }^{102}$ Viele Juden wurden noch am 24. Mai verhaftet und in Lager außerhalb der Hauptstadt transportiert. Vor allem hatte man es auf die Spitzen der jüdischen Gemeinschaft abgesehen, die vom Kommissariat noch in derselben Nacht ins Lager nach Samovit geschafft wurden. Einige konnten sich jedoch mit Hilfe von Bulgaren verbergen. Der mutige Rabbi Cion, Initiator zahlreicher jüdischer Proteste, wurde auf der Rückkehr von der Besprechung mit dem Metropoliten verhaftet und ebenfalls ins Lager geschickt. Der Oberrabbi Hananel dagegen durfte als Träger eines militärischen Verdienstordens in der Hauptstadt bleiben. Trotz der Protestaktionen der Öffentlichkeit war es also zu Internierungen von Juden gekommen. Immerhin wurde dem Metropoliten und anderen Prominenten von Regierungsseite zugesichert, daß die Juden nicht außerhalb Bulgariens gebracht würden. Die am 24. Mai und in den folgenden Tagen verhafteten Juden wußten nichts von der Kursānderung der Regierung und befürchteten, daß diese Aktionen der erste Schritt zur Deportation nach Polen seien. Vom Lager Samovit aus sahen sie mit Schrecken leere Schiffe im dortigen Donauhafen anlegen. ${ }^{103}$

Die Regierung plante zunāchst, die Sofioter Juden in etwa 20 Provinzstädten (aus Sicherheitsgründen nicht in Grenzorten, ausgenommen die Donaustädte) anzusiedeln. In den folgenden Wochen wurde die Masse der Sofioter Juden zum Verlassen der Hauptstadt aufgefordert. Sie hatten 1 1/2 Tage Zeit, der Aufforderung Folge zu leisten, andernfalls drohte ihnen die gewaltsame Ausweisung. Laut deutschen Berichten verließen in den folgenden Wochen 90 Prozent der Juden die Hauptstadt freiwillig und wurden in den von der Regierung vorgesehenen Stādten untergebracht. Einige tausend Juden konnten aufgrund persōnlicher Beziehungen in Sofia bleiben. Die Ausgewiesenen wurden in der Provinz den ōrtlichen Konsistorien anvertraut, die auch für deren Unterhalt aufzukommen hatten. Viele wurden zunächst in leerstehenden Schu-

100 Bericht Hoffmanns vom 7. Juni 1943 (486350-356).

101 Chary, Bulgarian Jews, S. $146 \mathrm{f}$.

102 Zur Tätigkeit von Stants- und Parteichef Todor Živkov während des Zweiten Weltkrieges siehe Hans-Joachim Hoppe, Todor Shiwkow - Eine politische Biographie, in: Osteuropa S (1978), S. 399-408, hier S. 399 f.

103 Chary, Bulgarian Jews, S. 148-151. 
len untergebracht, doch war dies nur kurze Zeit möglich, nämlich bis zum Ende der Frühjahrsferien. Das Eigentum der ausgewiesenen Sofioter Juden wurde versteigert. 104

Weiteren deutschen Forderungen (insbesondere des RSHA) nach Deportation bulgarischer Juden hielt der Gesandte Beckerle die besondere Situation in Bulgarien entgegen. In seinen Berichten warb er in Berlin um Verstāndnis für die bulgarische Haltung. Sogar Polizeichef Hoffmann schrieb nach Berlin, man solle die bulgarische Regierung nicht zu sehr unter Druck setzen, wenn man sie sich nicht zum Feind machen wolle. Grundsātzlich sei Sofia mit einer Lōsung im deutschen Sinne einverstanden, doch die Hauptschwierigkeit sei, ${ }^{\mathrm{daß}}$ die bulgarische Regierung diese Frage nicht einfach über das Knie brechen kann“. Sie müsse auf innen- und außenpolitische Faktoren Rücksicht nehmen. Beckerle bekräftigte diesen Eindruck, Boris und Filov hātten ihm gegenüber geāußert, die Juden würden dringend für den Arbeitseinsatz in Bulgarien benōtigt. Die Deutschen müßten auch die bulgarische Mentalitāt berücksichtigen, den Mangel an „ideologischer Stärke“, ihr Unverstāndnis gegenüber Antisemitismus. Beckerle schloß seinen Bericht mit der Bemerkung, nur ein deutscher Sieg könne an der bulgarischen Haltung in der Judenfrage etwas ändern. ${ }^{105}$ Deutsche Erfolge aber blieben aus, vielmehr kam es zu weiteren Rückschlägen an der Ostfront. Waren im Mārz und im Mai 1943 die Proteste der jüdischen Gemeinde und der Ö́ffentlichkeit in Bulgarien der auslösende Faktor zur Verhinderung von Deportationen und Vernichtung, so sorgten dann die Erfouge der Alliierten dafür, daß die bulgarische Regierung bei ihrem Beschluß blieb, die Juden nicht zu deportieren. 106

Der plötzliche Tod des Zaren Boris am 28. August 1943 fiel in die Zeit äußerer und innerer Krisen. Der Frontwechsel Italiens und die Rückschläge im Osten hatten das Ansehen der Achse auch auf dem Balkan geschwācht. Bulgariens Bedeutung als deutscher Verbündeter auf dem Balkan wuchs nach dem Ausfall Italiens. Die Führung übernahm ein dreiköpfiger Regentschaftsrat (Ministerpräsident Filov, der ehemalige Kriegsminister Michov und der Bruder des Königs, Prinz Kyrill), neuer Premier wurde der Senior des alten Kabinetts, Finanzminister Dobri Božilov. Sein Kabinett war das gemäßigste seit Januar 1940 - mit dem Exbotschafter in Ankara, Sava Kirov, als Außenminister, Ivan Beškov als Landwirtschaftsminister und Ivan Vazov als Handelsminister. Die beiden letzteren hatten den Protest gegen die Judenverfolgung unterzeichnet. 107

Innenminister wurde Dočo Christov, einer der führenden Antisemiten im Parlament und Freund des Ex-Innenministers Gabrovski. Neuer Kommissar für Judenfragen wurde Christo Stomaniakov, kein professioneller Antisemit wie Belev, sondern stellvertretender Staatsanwalt des Sofioter Appellationsgerichts. So erfolgte doch anläßlich des Personalwechsels ein Wandel in der Judenpolitik: angesichts des stārkeren deutschen Interesses an der Balkanregion verlegten die Bulgaren die Judenfrage auf eine höhere Ebene. 108 Der neue Innenminister war einer der führenden Antisemiten des Landes, nicht aber der Judenkommissar, so daß Ende 1943 faktisch der Druck auf

104 Ebenda, S. 151 f.; siehe auch den Bericht Hoffmanns vom 7. Juni 1943.

105 Siche Berichte Beckerles vom 7. Juni, 18. und 19. August 1943 (485357-359, K 206528-533); Hoffmann vom 24. Juni 1943 (173953-956); Wagner an Kaltenbrunner (RSHA), 31. August 1943 (K 206542-547).

106 Chary, Bulgarian Jews, S. $156 f$.

107 Zur neuen bulgarischen Führung ausführlich Hoppe, Bulgarien, S. 148 f.

108 Chary, Bulgarian Jews, S. 165 f. 
die Juden nachließ. Die juristischen Restriktionen blieben bestehen, wurden aber nicht verschärft; vor allem hörte die Debatte über Judendeportationen auf. In einem seiner ersten Erlasse befahl der neue Innenminister, das bewegliche Eigentum der aus Sofia ausgesiedelten Juden aufzulösen. Die Deutschen interpretierten dies als Bekräftigung einer von der Regierung eingeschlagenen Judenpolitik im deutschen Sinne. Doch wurde den Juden erlaubt, im November 1943 für zehn Tage in die Hauptstadt zurückzukehren, um ihre Eigentumsfragen zu regeln. ${ }^{109}$

Während dieser Monate lebten die Sofioter Juden zusammengedrāngt in den Hāusem der Provinzjuden; Personen im arbeitsfähigen Alter dienten getrennt von ihren Familien in Arbeitseinheiten. In der Provinz waren die Juden einem rigorosen Regime mit beschränkter Bewegungstreiheit und geringen Arbeitsmöglichkeiten ausgesetzt. Die ab August 1942 erlassenen Restriktionen blieben in Kraft Nur eine kleine Minderheit der Juden befand sich in den Konzentrationslagem in Pleven und Samovit. In größeren Lagern wurden hauptsächlich Kommunisten und Partisanen interniert. Unter ihnen waren auch einige Juden, die mit den Partisanen gekämpft hatten oder Mitglieder der Kommunistischen Partei waren.

Mit dem Vormarsch der Roten Armee schwand der deutsche Einfluß in Bulgarien: die Regierung schwankte zwischen den Mächten und wartete, bis der Kampf entschieden war. Die kommunistischen Partisanenaktivitāten nahmen Ende 1943 zu; die sowjetischen Forderungen eskalierten mit den Erfolgen an der Front. So protestierte die Sowjetunion gegen die Verletzung der Neutralitãt durch den deutschen Schiffsbau in Varna und drohte mit Kriegserklärung an Bulgarien; außerdem forderte sie die Wiedereröffnung von Konsulaten. Die deutsche Seite hingegen forderte erfolglos den Bruch mit der Sowjetunion. Im November 1943 und im Januar 1944 erfolgten alliierte Luftangriffe auf Sofia, so daß man mit der Evakuienung vieler Behörden begann. Ironischerweise mußten schließlich die Bulgaren - wie acht Monate zuvor die Juden - Sofia verlassen. Gleichzeitig suchte die Regierung Kontakte zu den Alliierten anzuknüpfen. Schon im Oktober 1943 ließ die antisemitische Propaganda mit Rücksicht auf die Alliierten nach; die Bereitschaft zu Aktionen gegen die Juden schwand.

Nach Bildung eines Kabinetts am 1. Juni 1944 mit neuen, teilweise dem Hof nahestehenden Personen und dem ehemaligen Landwirtschaftsminister Ivan Bagrjanov an der Spitze suchte die Regierung einen Ausweg aus dem Krieg im Kontakt mit den Alliierten. Ministerpräsident Bagrjanov und Kriegsminister Rusi H. Rusev sprachen mit prominenten Vertretern der jüdischen Gemeinschaft; der Premier kündigte eine neue Politik gegenüber den Juden an, doch müsse die Regierung vorsichtig sein, solange die Deutschen noch im Lande seien und die Gefahr einer Intervention bestehe. Schließlich ernannte Judenkommissar Stomaniakov den Obersten Avram Tadžer, den Juden und Bulgaren gleichermaßen schātzten, zum Vorsitzenden des Zentralen Konsistoriums. Er hatte im Ersten Weltkrieg mit Bagrjanov als Offizier in derselben Einheit gedient. 110 


\section{Das Ende der Judenverfolgung in Bulgarien}

In der Regierungserklārung vom 17. August 1944 kündigte Bagrjanov vorsichtig Bulgariens Ausscheiden aus dem Krieg, den Abzug der Deutschen und Erleichterungen in der Judenfrage im geeigneten Moment an. Während Stojčo Mošanov mit einer bulgarischen Delegation in der Türkei bei den Alliierten um Frieden bat, hob am 31. August 1944 das Kabinett die Judenrestriktionen auf, die durch das Gesetz zum Schutz der Nation, den Erlaß vom 26. August 1942 und durch andere Bestimmungen definiert waren. Die Juden erhielten ihre vollen bürgerlichen Rechte zurück; jüdische Organisationen und Institutionen wurden wieder zugelassen. Auch wirtschaftlich wurden die Juden den Bulgaren wieder gleichgestellt; die Sondersteuern wurden aufgehoben. So war die Rechtslage vor dem 29. Januar 1941 wiederhergestellt. Auch versprach die Regierung die Rückgabe des konfiszierten Eigentums; das KEV, dem Justiz- und Finanzministerium unterstellt, hatte nun die Aufgabe, zur Wiedereinsetzung der Juden in ihre alten Rechte beizutragen. Die Waffenstillstandsverhandlungen wurden inzwischen in Ägypten fortgesetzt; die Deutschen bereiteten ihren Abzug aus Bulgarien vor, nachdem sie Plāne einer Intervention oder der Einsetzung einer Rechtsregierung fallengelassen hatten, zumal die Rote Armee schon an der Donau stand.111

Am 2. September 1944 wurde eine deutlich westlich orientierte Regierung unter Konstantin Muraviev und aus Mitgliedern der ehemaligen legalen Opposition gebildet, gegenüber der man größeres Entgegenkommen der Alliierten erhoffte. Jedoch kamen die Waffenstillstandsverhandlungen in Kairo nicht voran. In der Judenpolitik erließ die neue Regierung nur wenige Durchführungsbestimmungen zu der vom Kabinett Bagrjanov beschlossenen Gesetzgebung. Erst am 7. September 1944 wurde den Juden, die gegen antisemitische Gesetze verstoßen hatten, eine Amnestie gewährt; viele Juden begannen nach Sofia zurückzukehren. ${ }^{112}$

Am 5. September 1944 erklärte die Sowjetunion Bulgarien den Krieg, um einen Vorwand zum Einmarsch zu haben, wāhrend die Vaterlāndische Front und Partisanen in der Provinz und am 9. September in Sofia die Macht übernahmen. Ein neuer Regentschaftsrat und eine neue Regierung unter Kimon Georgiev wurden gebildet. Am 28. Oktober 1944 wurde in Moskau der Waffenstillstand unterzeichnet. Danach war Bulgarien zur Aufhebung der diskriminierenden Gesetzgebung gegen die Juden verpflichtet ${ }^{113}$ Bulgarien wurde einer Alliierten Kontrollkommission unterstellt, die die Durchführung der Bestimmungen überwachen sollte. Die Regierung der „Vaterlāndischen Front" erfüllte endlich, was die Bagrjanov-Regierung dekretiert hatte: Am 23. September 1944 kündigte Propagandaminister Dimo Kazasov die Rückgabe jüdischen Eigentums an, was nicht leicht zu realisieren war, da die Wirtschaftslage Bulgariens kritisch und das jüdische Eigentum hãufig verloren oder zerstōrt war. Die Politik der Verstaatlichung von Industrie und Handel stellte abermals die Rückgabe des Ei-

111 Ausführtich Hoppe, Bulgarien, S. 158-174; Chary, Bulgarian Jews, S. 173 ff.

112 Hoppe, Bulgarien, S. 175-180; Chary, Bulgarian Jews, S. $175 \mathrm{f}$.

113 Der Waffenstillstand mit Bulgarien vom 28. Oktober 1944 ist abgedruckt in: Sovetsko-bolgarskie otnosenija 1944-1948gg, Dokumenty i Materialy, Moskve 1969, S. 32-42; in Englisch in United Nations, Amistice Agreement between USA, USSR, UK and Bulgaria, Washington 1945. In deutscher Sprache ist der Waffenstillstand aufgrund eines Funkberichts vom 29. Oktober 1944, Sonderzug. Waffenstillstandsbedingungen für Bulgarien, enthalten in: PA, Handakten Ritter, Bulgarien, Bd. 1-3 (358011-017). 
gentums in Frage. Doch versprach die Regierung finanzielle Entschädigung, soweit sie dazu in der Lage war. So war Hilfe von außen nötig: Ben Gurion sicherte Ende 1944 bei seinem Besuch in Bulgarien den bulgarischen Juden Hilfe zu; auch sprang das Jewish Joint Distribution Committee zugunsten armer Juden ein. ${ }^{114}$ 1945/46 wurden Prozesse gegen die für die Kriegspolitik Verantwortlichen geführt. Die mit der Ausführung der Judenpolitik betrauten Personen, insbesondere die Mitarbeiter des KEV standen ab Mărz 1945 vor Gericht.

Doch wichtiger als Entschādigungen oder Prozesse war ab 9. September 1944 für die bulgarischen Juden die Frage, ob sie in Bulgarien bleiben oder nach Palästina auswandern sollten. Die Zionisten propagierten die Ubersiedlung nach Palāstina, Kommunisten und Linke zunāchst das Verbleiben der Juden in Bulgarien. Erst als sich die Beziehungen zwischen Großbritannien und der Sowjetunion verschlechterten und die Kommunisten im Oktober 1946 unter Georgi Dimitrov den Regierungskurs allein bestimmten, ließ der Widerstand gegen eine jüdische Emigration nach. Bereits 1946 und 1947 verließen viele Juden Bulgarien und siedelten nach Palästina über. Doch die britische White-Paper-Policy limitierte die jüdische Immigration. Wie alle Ostblockstaaten unterstützte auch Bulgarien den Plan eines jüdischen Staates und das Teilungsprojekt der UN für Palāstina von 1948. Als die Zionisten den souverānen Staat Israel proklamierten, erkannte ihn die bulgarische Regierung an. Die Dimitrov-Regierung erlaubte den Juden die freie Auswanderung nach Israel, die im Mai 1948 verstärkt einsetzte. 40000 Juden verließen Bulgarien und siedelten in Israel. Diesc erstaunliche Aktion diente der Stärkung des israelischen Staates; sie sollte wohl die Briten brüskieren, den bulgarischen Staat von seiner Pflicht zur Rückgabe jüdischen Eigentums weitgehend befreien und aus Bulgarien einen ethnisch homogeneren Staat schaffen. ${ }^{115}$

Heute leben in Bulgarien ca. 5500 Juden, davon allein 3200 in Sofia, 600 in der zweitgrößten Stadt Plovdiv und die restlichen in verschiedenen Städten wie Varna, Burgas, Ruse, Jambol, Stanke Dimitrov und Kjustendil. Gesellschaftlich werden sie von der "Jüdischen Kulturorganisation" in Sofia vertreten.

\section{Bilanz}

Alle bulgarischen Juden, ca. 50000, wurden gerettet; sie überstanden den Zweiten Weltkrieg unversehrt, obwohl Bulgarien mit Hitler-Deutschland verbündet war. Die von den Deutschen geforderten Maßnahmen gegen die Juden waren bei den Bulgaren unpopulār. Seit Jahrhunderten gewohnt, mit anderen Nationalitāten wie Türken, Griechen, Zigeunern, Armeniern und Juden zusammenzuleben, fehlte der bulgarischen Bevölkerung das Verständnis für Antisemitismus. Nur einige Splittergruppen auf der Rechten wie Ratnici und Legionäre gaben sich militant antisemitisch.

Die bulgarische Führung übernahm die deutsche Judengesetzgebung nur halbherzig und rudimentär; ihre Judenpolitik war mehr Teil ihres Taktierens, nämlich ihre

114 Chary, Bulgarian Jews, S. 178.

113 Hans-Joachim Hoppe, Georgi Dimitroff und die bulgarischen Juden, in: Algemeine Jüdische Wochenzeitung vom 19. Juli 1974; ausführlich Oschlies, Bulgarien, S. 90-94. 
Loyalitāt gegenüber Deutschland unter Beweis zu stellen, solange von diesem Schutz des Landes gegenüber der Sowjetunion und Erfüllung territorialer Wünsche zu erwarten waren. Hauptmaxime der Politik der bulgarischen Führung und insbesondere des Königs waren āußere Sicherheit und innere Stabilität. So kamen dem Kriegsverlauf und der öffentlichen Meinung Bulgariens auch bei der Judenpolitik großes Gewicht zu. Als im Mārz und dann noch einmal im Mai 1943 die Frage der Deportation der bulgarischen Juden zur Debatte stand und die Vorbereitungen schon anliefen, war neben den Protesten aus der Öffentlichkeit entscheidend, daß die bulgarische Führung in dieser Frage wegen der geschwächten deutschen Position nach Stalingrad auch Rücksicht auf die Alliierten nehmen mußte. Nach dem Sommer 1943 war die Gefahr für die Juden weitgehend gebannt, da die Deutschen sich mit dem ${ }_{n}$ Aufschub ${ }^{\text {“ der }}$ Deportationen zufrieden gaben und die Bulgaren im Hinblick auf ihre Sondierungen mit den Alliierten ihre Judenpolitik sogar schrittweise lockerten. Das Schicksal der Juden hātte nur noch durch einen Rechtsputsch, der aber selbst von den Deutschen als aussichtslos betrachtet wurde, und eine mögliche Okkupation des Landes, die anders als in Ungarn inopportun erschien, gefährdet werden kōnnen.116

Für die Rettung der bulgarischen Juden in der kritischen Phase des Frühjahrs 1943 war außer der Kriegslage der massive Protest der bulgarischen Gesellschaft wichtig. Die Proteste der Opposition hatten aber nur begrenzte Wirkung. Die kommunistische Agitation fand wegen der Randstellung der Partei kaum Beachtung. Prominente Oppositionspolitiker wie Mušanov, Stajnov und Petrov griffen die Judenpolitik als willkommenes Thema des politischen Kampfes gegen die Regierung auf und appellierten an das Gewissen der Öffentlichkeit. Entscheidend für die Umstimmung der Regierung waren jedoch die Proteste aus den eigenen Reihen: Die überraschende Aktion des Parlaments-Vizepräsidenten Pešev löste eine Serie von Protesten aus - der Parlamentarier, des Heiligen Synods, aus Kreisen des Hofes und gewichtiger Stimmen der Oberschicht, die bei der bulgarischen Führung und dem König Gehör fanden. Von Bedeutung waren nicht zuletzt die Aktivitāten der Juden selbst zu ihrer Rettung. Zur Änderung der Regierungspolitik und Verhinderung der Deportationen trug die Publizität, die die Frage der bulgarischen Juden, inzwischen selbst in höchsten Kreisen des westlichen Auslands, in Großbritannien und den USA errungen hatte, entscheidend bei.

Die bulgarischen Juden wurden gerettet, nicht hingegen die mazedonischen und thrazischen Juden. Insgesamt wurden mindestens 11343 Personen jüdischer Herkunft von den Bulgaren deportiert. Als Entschuldigung wird immer wieder angeführt, die bulgarische Regierung habe die Gebiete Mazedonien und Thrazien von den Deutschen geschenkt bekommen und daher dort nur eingeschränkte Vollzugsgewalt gehabt. Die Deportationen von dort seien deshalb im Grunde das Werk der Deutschen. Tatsache ist aber, daß Bulgarien sich die neuen Territorien trotz deutscher Vorbehalte einer endgültigen späteren Regelung im Rahmen der ${ }_{\text {NNeuen Ordnung }}$ administrativ voll einverleibt und in der Praxis entsprechend behandelt hat. Der Deportation stimmte die bulgarische Regierung zwar auf deutschen Druck, aber aufgrund eigener

116 Ungarn konnte sich in den Kriegsjahren ertolgreich den deutschen Wünschen nach Judendeportationen widersetzen, bis es im Mäz 1944 von deutschen Truppen besetzt und zu einer Regierungsumbildung veranlaßt wurde. Zur Judenpolitik und den Deportationen nach dem Frühjahr 1944 siehe den Beitrag über Ungarn im vorliegenden Band. 
unabhāngiger Entscheidung zu; auf ihre Weisung wurde ein Judenkommissariat eingerichtet; es waren bulgarische Beamte, die in Zusammenarbeit mit deutschen „Bera-

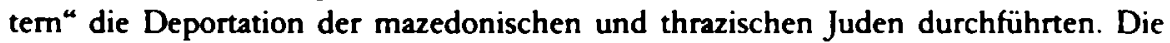
Bereitwilligkeit der bulgarischen Führung ist aus ihrem Ziel zu erklāren, den slawischen Bevōlkerungsanteil in den besetzten Gebieten durch Abwanderung der Griechen und Deportation der Juden zu erhöhen. Bei der Behandlung der mazedonischen und thrazischen Juden kannten die Bulgaren keine Skrupel, denn es waren nicht ihre "eigenen“, sondern "fremde“ Juden. Ihr Schicksal fand weder Fürsprecher, noch die Publizität, die den bulgarischen Juden das Leben rettete. Bulgarien war mit seiner Judenpolitik unter den Achsenländern ein Sonderfall, aber nicht die einzige Besonderheit.117 Bulgarien ist das Paradebeispiel für die Haltung eines Landes, das weder faschistisch noch antisemitisch, wohl aber "opportunistisch“ war.

Wie die Juden in kollaborierenden Ländern wie Bulgarien, Rumānien und Ungarn größere Chancen als in den besetzten Ländern hatten, so war für ihr Schicksal die Fürsprache aus Regienungskreisen wichtiger als die Aktivitāt der Opposition. Letztlich hing aber die Judenpolitik stärker von der äußeren als von der inneren Entwicklung ab. Noch 1942 wuchs der deutsche Druck auf Bulgarien und war auch im März 1943 trotz der Rückschläge an der Ostfront bestimmend, so daß die Regierung in Bulgarien einen Kompromiß einging und die Juden aus den besetzten Gebieten deportierte. Es scheint, als ob die mazedonischen Juden zugunsten der in Altbulgarien geopfert wurden. Doch spielte Zar Boris offenkundig auf Zeit, überließ die Juden in Mazedonien den Deutschen und erfand immer neue Gründe, um die bulgarischen Juden zurückzuhalten. Als im Mai 1943 die Juden aus dem bulgarischen Kemland deportiert werden sollten, war der deutsche Einfluß durch den Krieg schon so geschwächt, daß sich die Opposition gegen die Judenpolitik durchsetzte. Und ab Sommer 1943 überwogen Rücksichten auf die innere öffentliche Meinung und auf die Allierten, mit denen man einen Sonderfrieden anstrebte, die Beachtung deutscher Wünsche. So wurden die bulgarischen Juden gerettet. ${ }^{118}$

117 Ausführlich Chary, Bulgarian Jews, S. 193-199.

118 Oschlies, Bulgarien, S. $77 \mathrm{f}$. 